\title{
Valuing portfolios of interdependent real options using influence diagrams and simulation-and-regression: A multi-stage stochastic integer programming approach ${ }^{\text {th }}$
}

\author{
Sebastian Maier ${ }^{\mathrm{a}, *}$, John W Polak ${ }^{\mathrm{b}}$, David M Gann ${ }^{\mathrm{c}}$ \\ ${ }^{a}$ Department of Civil and Environmental Engineering and Grantham Institute - Climate Change and \\ the Environment, Imperial College London, London SW7 2AZ, UK \\ ${ }^{b}$ Centre for Transport Studies and Urban Systems Laboratory, Imperial College London, London SW7 \\ 2AZ, UK \\ ${ }^{c}$ Imperial College Business School, Imperial College London, London SW7 2AZ, UK
}

\begin{abstract}
Although real options generally occur within portfolios, most valuation approaches based on either option pricing or decision analysis alone focus on single well-defined options. In this paper we present a new approach for modelling and approximating the value of portfolios of interdependent real options using both influence diagrams and simulation-and-regression. The key feature of this approach is that it translates the interdependencies between real options into a set of constraints and then directly models the dynamics of all underlying uncertainties using (Markovian) stochastic processes. These are then integrated in a portfolio optimisation problem which is formulated as a multi-stage stochastic integer program. Applying a simulation and regression approach to approximate the value of this optimisation problem, we present a transparent valuation algorithm that explicitly takes into account vector-valued exercise decisions and the state variable's multidimensional resource component. The approach is therefore applicable to a wide range of complex investment projects with both inherent interdependent flexibilities and many underlying uncertainties. The approach is illustrated by evaluating a complex natural resource investment that features both a large portfolio of interdependent real options and four stochastic factors. We analyse the way in which the approximated value of the portfolio and its individual options are affected by the initial copper price as well as by the degrees of production cost and copper price uncertainty.
\end{abstract}

\footnotetext{
This paper is a significantly expanded version of a paper first presented at the 19th Annual International Real Options Conference in Athens and Monemvasia, Greece, in June 2015.

${ }^{*}$ Corresponding author. Tel.: +44 (0)20 7594 2705; fax: +44 (0)20 75946102.

Email addresses: sebastian.maier@imperial.ac.uk (Sebastian Maier), j.polak@imperial.ac.uk (John W Polak), d.gann@imperial.ac.uk (David M Gann)
} 
Keywords: Real options portfolio, Influence diagram, Simulation-and-regression, Approximate Dynamic Programming, Natural resource investment

\section{Introduction}

Making sound investment decisions in the context of uncertainty and irreversibility is a challenging problem for decision makers in both the public and private sectors. The value of an investment project and its exposure to risks are generally affected directly by both a sequence of decisions, which may be strategic, operational or tactical (ChevalierRoignant et al., 2011), and their interactions (e.g. between financing and investment decisions (Myers, 1974)). In addition, decisions makers often have to make such sequential decisions simultaneously with respect to several interacting projects, thus have to manage portfolios of investment projects, rather than individual ones.

While there exist a range of capital budgeting techniques, valuation methods based on simple temporal discounting are still widely used in practice (Ryan and Ryan, 2002 , Bennouna et al., 2010). Given their prevalence, discounting cash flow (DCF) methods such as net present value (NPV) have been extended considerably in order to allow for more accurate investment analyses. Popular extensions include the Weighted Average Cost of Capital, or WACC; the Capital Asset Pricing Model, or CAPM; Monte Carlo simulation; and sensitivity analysis. Despite all these extensions, however, the inherent limitation of DCF methods remains: since they assume that investment decisions are "now or never" propositions they do not correctly take into account the value of managerial flexibility, thereby undervalue uncertain investment projects.

To address this limitation, real options analysis (ROA), or real options valuation, has received increasing attention and a wide range of different ROA approaches and techniques have been developed (De Reyck et al., 2008). In particular, ROA has extended to accommodate portfolios of interdependent real options (Trigeorgis, 1995), potentially significantly enhancing the applicability of ROA to many practical situations. However, recent surveys indicate that the practical application of ROA is still limited, and largely confined to companies and industries with more sophisticated analytical cultures (Block, 2007) or those exposed to high levels of financial uncertainty (Verbeeten, 2006). Lander and Pinches (1998) highlighted a number of institutional factors impeding the practical application of ROA techniques and Zapata and Reklaitis (2010) noted a number of theoretical limitations in ROA when used in a portfolio context. A key limitation is that when ROA is extended to the context of portfolios of options, traditional option valuation techniques (e.g. binomial/lattice and finite difference) become impractical 
(Longstaff and Schwartz, 2001; Gamba, 2003; Trigeorgis, 2005).

In this paper we present a new approach to model and approximate the value of portfolios of interdependent real options using influence diagrams and simulation-andregression. Although it is rarely used by real options analysts, the influence diagram (ID) is a promising alternative to the traditionally-applied decision tree and has many advantages as a framework for identifying, defining, and modelling interdependent flexibilities in investment projects. For example, IDs are intuitive and can be readily applied by decision makers to identify flexibilities (Lander and Shenoy, 1999), thereby focusing on the decisions the manager can make, rather than the risk modelling (Sick and Gamba, 2010). From a modelling perspective, IDs allow a more compact representation than lattice/tree techniques (Charnes and Shenoy, 2004), particularly in situations where there are multiple uncertainties and a sequence of decisions, or path dependency (Demirer et al. 2003). This is because IDs do not scale with the number of uncertainties and grow linearly rather than combinatorially in the number of decision variables considered (Lander and Pinches, 1998). Finally, ID representations are simple, intuitive, transparent and flexible. In our approach, we use IDs to graphically model the flexibilities contained in a portfolio of interdependent real options. The interdependencies between options are then translated into a set of constraints and integrated into the portfolio optimisation problem, which is formulated as a multi-stage stochastic integer program.

In order to approximate the value of portfolios of interdependent real options we apply simulation combined with parametric regression. It is widely acknowledged that simulation techniques, despite their computational complexity, have significant advantages over traditional option pricing techniques such as analytical and lattice-based methods (Pringles et al. 2015). Several authors have proposed numerical methods for the valuation of single American options using simulation and regression (Denault and Simonato, 2017); Stentoft (2014) recently compared the approaches of Carriere (1996); Tsitsiklis and Van Roy (2001); Longstaff and Schwartz (2001) and recommends the least squares Monte Carlo (LSM) approach of Longstaff and Schwartz (2001) for computational reasons. While the portfolio optimisation problem is formulated here as a multi-stage stochastic program (rather than an optimal stopping problem for American option pricing), we also apply "continuation function approximation" 1 by using both a parametric model and a least-squares method ${ }^{2}$ like Tsitsiklis and Van Roy (2001);

\footnotetext{
${ }^{1}$ It is important to note that functional approximations have been used at least since Bellman and Dreyfus (1959) to increase the computational efficiency of dynamic programming.

${ }^{2}$ By contrast, Tsitsiklis and Van Roy (1999) use a stochastic approximation algorithm to fit the model.
} 
Longstaff and Schwartz (2001). In this way, we are able to approximate the complex (hard or impossible to determine) continuation functions that describe the expected future contributions associated with transitions in the ID. These approximations are then used in the simulation-and-regression-based valuation algorithm to determine pathwise optimal decisions - by solving integer programs - for all available transitions at each possible resource state, subject to the constraints that describe interdependencies. We refer to this method as the influence diagram simulation-and-regression (IDSR) approach.

The main contributions of this work are: (i) introduction of an approach for the valuation of portfolios of interdependent real options using IDs and simulation-andregression, where IDs are used to graphically model the interdependencies between real options, which are then translated into a set of constraints; (ii) modelling of the portfolio optimisation problem as a sequential (stochastic) decision problem with vector-valued exercise decisions and formulation as a multi-stage stochastic integer program; (iii) development of a simulation-and-regression-based valuation algorithm that consists of a forward and backward induction procedure and that contains several important features specific to option portfolios such as the explicit consideration of the state variable's multidimensional resource component; and (iv) a demonstration of the ability of the proposed approach to evaluate a complex natural resource investment that features both a large options portfolio and four underlying uncertainties, including an investigation of the way in which the value of the portfolio and its individual real options are effected by both the underlying copper price level and the degrees of different uncertainties as well as an analysis of the effect of different parametric models on the value of the portfolio. These four areas of innovation are important as they highlight the potential of our approach to enhance the applicability of ROA to a wide range of complex yet practical and important investment problems and to lay the basis for further theoretical developments.

The remainder of this paper is organized as follows: Section 2 reviews the relevant literature on portfolios of real options. Section 3 describes both the approach that a decision maker can apply to model inherent flexibilities and formulates the corresponding portfolio optimisation problem. A valuation algorithm is presented in Section 4 that approximates the optimal value of portfolios of interdependent real options (Subsection 3.2. The approach is then applied to the example of evaluating a complex natural resource investment in Section 5. Section 6 presents and discusses the results. Finally, Section 7 provides some concluding remarks. 


\section{Literature review}

Early studies on portfolios of real options explored the concept rather qualitatively and in the context of corporate strategy and planning (Myers, 1984: Trigeorgis and Kasanen, 1991; Bowman and Hurry, 1993; Luehrman, 1998; Smit and Trigeorgis, 2006; Anand et al., 2007). Trigeorgis (1993) investigated the nature of interactions between a firm's real options and found that options' individual values are generally non-additive. More recently, Trigeorgis (2005) argued that decision problems represented by a portfolio of interdependent real options can be decomposed into a few basic-building blocks (i.e. individual options) and then combined by one of four commonly encountered basic decision operators, which are "or" ( $\max$ ), "and", "average" and "multi-stage" (compound).

Various scholars have presented approaches to value portfolios of real options in the context of specific practical applications. One of the first publications to do so was Rose (1998), who valued two interacting options embedded in a toll-road project and found that ignoring the options' interactions results in significantly underestimated project values. Other relevant articles presented approaches in the context of $\mathrm{R} \& \mathrm{D}$ projects (Vassolo et al., 2004; McGrath and Nerkar, 2004; van Bekkum et al., 2009; Zapata and Reklaitis, 2010), maritime investments (Bendall and Stent, 2007), IT investments (Pendharkar, 2010), and transmission network expansion (Loureiro et al., 2015). While these approaches have applied ROA in a portfolio context, they have developed rather inflexible and restricted quantitative approaches tailored to specific applications and limited to problems instances with specific features in terms of both options portfolio and uncertainties; by contrast, this paper takes a fundamentally different approach by proposing a valuation approach for portfolios of interdependent options applicable to a wide range of complex and risky investment problems in many practical situations.

A number of publications aimed at presenting more general quantitative frameworks for the problem of valuing portfolios of real option $3^{3}$. In an important early theoretical contribution, Childs et al. (1998) provided closed-form (analytic) solutions for the value of two investment project that can be developed either in parallel or in sequence. Smith and Thompson (2008) provided analytic solutions whilst considering the problem of valuing a portfolio of sequencing options that represent projects. Considering one underlying source of uncertainty, Meier et al. (2001) proposed two models: the first combines contingent claims analysis (replicating portfolios) with the well-known Knapsack problem to select projects, whereas the second is essentially an "Asset Liability Model"

\footnotetext{
${ }^{3}$ Another related article is Zhang and Babovic (2011), which integrates evolutionary algorithms into a real options framework to value and select portfolios of real options.
} 
solved by stochastic programming whilst combining simulation with a binomial tree for scenario generation. Despite presenting more general portfolio-based real options approaches, neither addresses the problem in such a general way as we do because their focus is rather on portfolios of interrelated projects than on interdependent real options. Furthermore, these approaches are simply impractical in most real-life situations.

Several recent studies have explored more holistic approaches including Gamba (2003); Wang and de Neufville (2004); Brosch (2008). Most recently, the work of Brosch (2008) addressed portfolios of real options by proposing a forward-backward looking algorithm based on stochastic mixed-integer programming and lattice/tree modelling, where the forward looking element captures the budget constraint by making sure only feasible paths can be chosen from. The framework presented by Wang and de Neufville (2004) consists of an options identification stage which contains a screening and simulation model, plus an options analysis stage which applies a stochastic mixed-integer programming model and a binomial technique for scenario generation. Interestingly, both authors discussed computational issues with respect to the optimality of solutions, but only Brosch (2008) also discussed simulation, yet dismissed it as an alternative technique in his model. While these studies constitute important contributions, for example the global, dynamic budget constraints in Brosch (2008) as well as the identification and definition of real options in physical systems by Wang and de Neufville (2004), the combined complexity of applying both binomial techniques for scenario generation and stochastic mixed-integer programming for optimal options timing as well as the resulting adverse computational issues make both of them impractical in most real-life situations.

In contrast, the framework presented by Gamba (2003) overcomes the computational limitations of the binomial techniques used in Wang and de Neufville (2004) and Brosch (2008) by applying simulation and linear regression. In fact, Gamba considered a portfolio of interdependent real options that can be decomposed into a set of simple real options, which can be independent, mutually exclusive, compound, or of the switching type, and presented decision rules for each of the four cases. These rules were then used within a valuation procedure that applies the LSM algorithm to analyse the four sub-problems individually. Although Gamba has proposed an interesting extension of the LSM algorithm, his paper neither addresses the modelling of portfolios of interdependent real options, nor presents a single framework (it considers four sub-problems individually). Our work differs in a number of ways when compared with (Gamba, 2003 , Wang and de Neufville, 2004, Brosch, 2008). For example, we use IDs to model portfolios of interdependent real options and translate the interdependencies into constraints. Furthermore, we integrate both the constraints and the directly modelled dynamics of all 
underlying uncertainties into a multi-stage stochastic integer program in which the continuation function is approximated by parametric regression. In order to illustrate the IDSR approach, we apply it to the evaluation of a complex natural resource investment.

\section{Problem formulation}

In this section, we present our approach to both the modelling of portfolios of interdependent real options and the formulation of the related portfolio optimisation problem as a multi-stage stochastic integer program.

\subsection{Modelling flexibilities with influence diagrams}

We consider the valuation of an investment project that is represented by a portfolio of interdependent real options. The flexibilities contained in this portfolio of interdependent real options are then modelled through an ID, which is composed of both a graphical and a numerical part. The former consists of two elements: a set of (decision and terminal) nodes $\mathcal{N}=\{1,2, \ldots, N\}$, which may represent stages of development or operating modes, as well as a set of directed edges $\mathcal{H}=\{1,2, \ldots, H\}$, which represents the transitions linking the nodes in the ID, or in other words the flexibilities available to the decision maker. Unlike most modelling approaches for IDs in the context of real options (e.g. see Lander and Pinches (1998); Lander and Shenoy (1999); Charnes and Shenoy (2004), we allow for cycles in the ID, which is then represented through a directed cyclic graph $(\mathcal{N}, \mathcal{H})$ instead of an acyclic one. Although we only consider decision nodes, so apply the deterministic use case of IDs as noted by Howard and Matheson (2005), our specification can be easily extended to the probabilistic case by including chance nodes in the ID, as shown by Charnes and Shenoy (2004). Appendix A contains an example of an American option to illustrate the graphical part of the modelling approach.

The numerical part of the ID is specified by information associated with both nodes and transitions. Let the state of the system at time $t, S_{t}$, be composed of a resource and an information component denoted by $R_{t} \in \mathcal{R}_{t}$ and $I_{t} \in \mathcal{I}_{t}$, respectively, thus having $S_{t}=\left(R_{t}, I_{t}\right)$, where $\mathcal{R}_{t}$ and $\mathcal{I}_{t}$ are the corresponding resource and information state spaces. In general, $R_{t}$ is an endogenous component (evolves deterministically), whereas $I_{t}$ is an exogenous component (evolves stochastically). The former is modelled to contain at least information about the current decision node $N_{t} \in \mathcal{N}$, but, generally, will contain further problem-dependent resource state variables. On the other hand, $I_{t}$ contains one or several stochastic factors (or random variables) describing the value of the problem's uncertain parameters at $t$. Most existing real options valuation approaches consider $I_{t}$ 
to represent the "state" and do not explicitly model $R_{t}$. The few exceptions usually only consider either a discrete (Nadarajah et al. 2017) or continuous (Denault et al. 2013) scalar for $R_{t}$. Here, in order to deal with complexities of portfolios of real options, including path-dependencies and interdependencies between options, we explicitly consider $R_{t}$ to be a vector made up of multiple resource state variables characterising the valuation problem 4 .

In order to simplify the valuation algorithm presented in the next section, we assume that $\mathcal{N}$ contains exactly one beginning node (no incoming transition(s)), but may have several terminal nodes, which are characterised through not having outgoing transitions. The value of a terminal node at $t$ is given by its terminal value $G_{t}^{T}\left(S_{t}\right)$, for all $S_{t} \in\left\{S_{t}^{\prime} \in\right.$ $\left.\mathcal{S}_{t}: b^{D}\left(N_{t}^{\prime}\right)=\emptyset\right\}$, where $b^{D}\left(N_{t}\right)$ represents the set of outgoing transitions of node $N_{t}$. The set of decision times, which is often referred to as decision epochs in the Markov Decision Process (MDP) literature, is denoted by $\mathcal{T}$.

With regard to the information associated with transitions, there are three elements to any transition $h \in \mathcal{H}$ :

1. The feasible region $\mathcal{A}_{S_{t}}$, which is composed of one or more constraints that describe the interdependencies between flexibilities, defines the transition(s) one can make given state $S_{t}$. Let the decision to make any transition $h \in b^{D}\left(N_{t}\right)$ at node $N_{t}$ be represented by a binary decision variable $a_{t h} \in\{0,1\}$, where $a_{t h}=1$ means transition $h$ is made at time $t$ and vice versa, as well as let the duration of transition $h$ be $\Delta_{h}$. Then, the vector $a_{t}=\left(a_{t h}\right)_{h \in b^{D}\left(N_{t}\right)}$ has to satisfy all constraints defined in $\mathcal{A}_{S_{t}}$, in other words $a_{t} \in \mathcal{A}_{S_{t}}$.

2. The transition function, which is generically written as $S^{M}\left(S_{t}, a_{t}, W_{t+\Delta_{h}}\right)$, describes the evolution of the state $S_{t}$ from time $t$ to $t+\Delta_{h}$ when making transition $h$ and given new exogenous information $W_{t+\Delta_{h}}$ that is learned between $t$ and $t+\Delta_{h}$. In terms of the state's two components, $S^{M}(\cdot)$ can be interpreted as a composition of both a resource transition function $S^{R}(\cdot)$ and an information transition function $S^{I}(\cdot)$ which describe individually the evolution of $R_{t}$ and $I_{t}$ to $R_{t+\Delta_{h}}$ and $I_{t+\Delta_{h}}$, respectively, when making transition $h$ at time $t$.

3. The immediate payoff $\Pi_{t}\left(S_{t}, a_{t}\right)$ is obtained at time $t$ when making decision $a_{t}=$

\footnotetext{
${ }^{4}$ For example, in the transmission network expansion planning problem considered by Loureiro et al. (2015) $R_{t}$ would contain the current network configuration, whereas in the pharmaceutical R\&D portfolio valuation problem studied by Zapata and Reklaitis (2010) $R_{t}$ would include the number of resources allocated to drugs within different portfolio configurations and their stages of development at time $t$.
} 
$\left(a_{t h}\right)_{h \in b^{D}\left(N_{t}\right)}$ given state $S_{t}$. Note that $\Pi_{t}(\cdot)$ depends only on variables whose value is known at time $t$, so is deterministic, and is being received at the beginning of the period $t$ to $t+\Delta_{h}$.

\subsection{Portfolio optimisation problem}

The problem of determining the optimal value of the portfolio of interdependent real options is formulated as a multi-stage stochastic integer program. Unlike the approach of Gamba (2003), who decomposed a portfolio of interacting real options into a set of independent, compound, mutually exclusive and switching options and then valued these sub-problems individually by enumeration, this paper proposes a single framework to value a portfolio of interdependent real options whilst using both a set of constraints and a vector of binary variables to model strategic interdependencies and exercise decisions, respectively. Let the optimal value of the portfolio of real options at time $t$ given state $S_{t}$ be denoted by $G_{t}\left(S_{t}\right)$. The value of the portfolio of interdependent real options, $G_{0}\left(S_{0}\right)$, is given by the optimal solution of the stochastic optimisation problem:

$$
G_{0}\left(S_{0}\right)=\max _{\left(a_{t}\right)_{t \in \mathcal{T}}} \mathbb{E}\left[\sum_{t \in \mathcal{T}} e^{-k t} \Pi_{t}\left(S_{t}, a_{t}\right) \mid S_{0}\right]
$$

where $S_{0}$ is the state at time $0, a_{t}=\left(a_{t h}\right)_{h \in b^{D}\left(N_{t}\right)}, a_{t} \in \mathcal{A}_{S_{t}}, a_{t h} \in\{0,1\}, k$ is the risk-free rate, and $S_{t+\Delta_{h}}=S^{M}\left(S_{t}, a_{t}, W_{t+\Delta_{h}}\right)$.

Applying Bellman's "principle of optimality", the above optimisation problem with objective (1) can be solved recursively by using the following value function for each state $S_{t} \in\left\{S_{t}^{\prime} \in \mathcal{S}_{t}: b^{D}\left(N_{t}^{\prime}\right) \neq \emptyset\right\}$ at time $t$ :

$$
\begin{array}{rlr}
G_{t}\left(S_{t}\right)=\max _{a_{t}} & \Pi_{t}\left(S_{t}, a_{t}\right)+\mathbb{E}\left[e^{-k \Delta_{h}} G_{t+\Delta_{h}}\left(S_{t+\Delta_{h}}\right) \mid S_{t}, a_{t}\right] \\
\text { s.t. } & a_{t} \in \mathcal{A}_{S_{t}}, & \\
& a_{t h} \in\{0,1\}, & \forall h \in b^{D}\left(N_{t}\right), \\
& S_{t+\Delta_{h}}=S^{M}\left(S_{t}, a_{t}, W_{t+\Delta_{h}}\right), & \forall h \in b^{D}\left(N_{t}\right),
\end{array}
$$

with the terminal condition $G_{t}\left(S_{t}\right)=G_{t}^{T}\left(S_{t}\right)$, for all $S_{t} \in\left\{S_{t}^{\prime} \in \mathcal{S}_{t}: b^{D}\left(N_{t}^{\prime}\right)=\emptyset\right\}, t \in \mathcal{T}$. Ultimately, the aim of solving the stochastic dynamic programming (SDP) recursion (2)-(5) is to determine a decision vector $a_{t}^{*}=\left(a_{t}^{*}\left(S_{t}\right)\right)_{S_{t} \in \mathcal{S}_{t}}$ for all $t \in \mathcal{T}$, i.e. an optimal policy, that maximises the value of the portfolio of interdependent real options given the state $S_{0}$ at time $0, G_{0}\left(S_{0}\right)$. It is important to note that, unlike traditional solution approaches for MDPs, the terminal condition used in (2)-(5) is not directly dependent on time $t$, but on whether the current node $N_{t}$ in the ID is a terminal node, which may 
or may not has to be reached at a certain $t \in \mathcal{T}$. In the context of real option portfolios, for instance, one may decide to irreversibly abandon a project at any point in time $t \in \mathcal{T}$, thereby reaching the corresponding terminal node perhaps well before $\max \mathcal{T}$.

At this point, it is important to note that while many approaches for real options valuation resort to an algorithmic strategy based on a dynamic programming representation, their original optimisation problems are often distinctly different. For example, valuing an American-style option entails solving an optimal stopping problem, which requires finding the stopping time that maximises the option's value. The corresponding SDP recursion 5 then characterises an optimal exercise policy, that is the stopping rule which solves the optimal stopping problem (Glasserman, 2003). While switching options can be valued by simply extending this SDP formulation (see, e.g., Gamba (2003); Cortazar et al. (2008); Tsekrekos et al. (2012)), solving the embedded optimal switching problem means determining the optimal switching policy rather than just the stopping policy. The valuation of options with early and multiple exercise features such as swing and storage options can be formulated as a discrete MDP and then be solved, in theory, using SDP to obtain an optimal exercise policy, as described by Nadarajah et al. (2017).

In contrast to these stochastic optimisation problems, we formulate the options portfolio problem as a multi-stage stochastic integer program to account for the complexities involved in valuing portfolios of interdependent real options. These complexities include path-dependencies, interdependencies between options, as well as higher dimensional decision vectors and resource state variables. Unlike the standard MDP formulation mentioned above, which assumes the decision and resource state variable are scalars, in our formulation the decision variable $a_{t}$ and the resource state variable $R_{t}$ are vectorvalued and multidimensional, respectively. Also, our feasible region $\mathcal{A}_{S_{t}}$ is defined by a set of constraints, rather than just a discrete action space as in the optimisation problems described above, where $\mathcal{A}_{S_{t}}$ represents either a binary choice or a fairly small set of discrete choices. Importantly, it can be shown that the multi-stage stochastic integer program (1) and solving the SDP recursion (2)-(5) are equivalent, e.g. see Powell (2011).

\subsection{Curses of dimensionality}

In general, solving the backward recursion in (2)-(5) can be computationally expensive, even intractable due to at least three curses of dimensionality: (i) the high dimensionality of the resource state space $\mathcal{R}_{t}$ and information state space $\mathcal{I}_{t}$; (ii) the

\footnotetext{
${ }^{5}$ Interestingly, unlike Tsitsiklis and Van Roy (2001), Longstaff and Schwartz (2001) neither presented the optimal stopping problem nor formulated the SDP recursion despite obviously addressing these.
} 
inability to (exactly) compute the conditional expectation in (2); and (iii) the highdimensionality of the decision vector $a_{t}$ and the feasible region $\mathcal{A}_{S_{t}}$. Of these, the curses related to both $\mathcal{I}_{t}$ and (ii) are being addressed through the simulation and parametric regression approach ${ }^{6}$ described in Section 4. Also, in simple real option problems the action and resource state spaces are, in general, relatively small so the dimensionality of $\mathcal{R}_{t}$ and (iii) can be neglected. For example, in the case of swing and storage options considered by Nadarajah et al. (2017), where both $a_{t}$ and $R_{t}$ are scalar discrete, the number of actions and $\mathcal{R}_{t}$ are relatively small. They can therefore assume that the intractability of their MDP (more precisely, of their SDP formulations) is solely due to the curses related to both $\mathcal{I}_{t}$ and (ii), and implicitly ignore the ones related to $\mathcal{R}_{t}$ and (iii).

In the context of real option portfolios, however, the action space or the resource state space or both will generally be larger, potentially making the related curse(s) difficult to ignore. In some practical option portfolio problems $a_{t}$ will still be a rather low-dimensional vector of binary variables and $\mathcal{A}_{S_{t}}$ will be small in size, so (iii) can be neglected. Also, although $R_{t}$ is generally a vector of discrete variables, as demonstrated by the problem considered in Section 5 , in which $R_{t}$ has 4 dimensions, in general by appropriately modelling the problem at hand and carefully choosing relevant parameters a large resource state space $\mathcal{R}_{t}$ is prevented and a computationally manageable valuation process is ensured. However, there are a range of practical problems that involve higher dimensional decision vectors and resource state variables as well as a larger number of constraint:7. Nevertheless, it should be relatively straightforward to extend our approach to account for higher dimensional problems by simply adapting the (already vector-valued) decision variabl $8 a_{t}$ and the (already multidimensional) resource state variable $R_{t}$ appropriately. Given the capabilities of modern integer programming packages, standard commercial solvers can then be used, again, to solve the embedded integer program within (2)-(5), and the dimensionality of $R_{t}$ can be addressed by suitable approximate dynamic programming approaches, e.g. see Denault et al. (2013).

\footnotetext{
${ }^{6}$ While such an approach is, in theory, not entirely free of the curse of dimensionality related to $I_{t}$ because the number of basis functions needed (e.g. multivariate polynomials) and the computational cost of estimating the parametric model's coefficients are not linear in the dimension of $I_{t}$ (Tsekrekos et al. 2012 ), the parametric model's size often remains very manageable in practice, e.g. see Judd (1998).

'For example, resource allocation problems - e.g. in facility location and power transmission planning - are naturally multidimensional and feature (very) large action and resource state spaces (Powell, 2011).

${ }^{8}$ It should be noted that our formulation lends itself to integer variables as binary variables can be used to represent general integers through binary expansion, and the feasible region can be adapted using standard modelling techniques associated with integer programming (Wolsey, 1998 Williams, 2013).
} 


\section{The valuation algorithm}

This section contains the approach to approximate the value of portfolios of interdependent real options as well as the corresponding simulation-based valuation algorithm.

\subsection{Approximating the continuation function by parametric regression}

The strategy chosen in this paper is to approximate the value of the conditional expectation in (2), which represents the continuation value, using a parametric regression model. Using such an approximation of the continuation value is a commonly used strategy in the Approximate Dynamic Programming literature (Tsitsiklis and Van Roy, 2001: Longstaff and Schwartz, 2001; Glasserman, 2003, Powell, 2011) and directly tackles the curse of dimensionality related to $\mathcal{I}_{t}$ and the outcome space. In particular, conditional upon being in state $S_{t}=\left(R_{t}, I_{t}\right)$ at time $t$ and making decision $a_{t} \in \mathcal{A}_{S_{t}}$, we approximate $\mathbb{E}\left[\cdot \mid S_{t}, a_{t}\right]$ of $(2)$ by the following finite-dimensional, continuous function:

$$
\hat{\Phi}_{t}^{L}\left(S_{t}, a_{t}\right)=\sum_{l=0}^{L} \hat{\alpha}_{l}\left(S^{R}\left(R_{t}, a_{t}\right)\right) \phi_{l}\left(I_{t}\right),
$$

where $L$ is the model's dimension, the functions $\left\{\phi_{l}\right\}_{l=0}^{L}$ are called basis functions (or features), and the optimal values of the coefficients (or weights), $\left(\alpha_{l}\left(S^{R}\left(R_{t}, a_{t}\right)\right)\right)_{l=0}^{L}$, are estimated using least-squares regression as described in the following subsection.

\subsection{The simulation-and-regression-based valuation algorithm}

To approximate the value of the multi-stage stochastic integer program, we apply a simulation-based algorithm that consists of both a forward and a backward induction procedure. The forward induction procedure initialises (and discretises) the state space $\mathcal{S}_{t}$ for all $t \in \mathcal{T}$. More specifically, using the numerical part of the ID and starting at $t=0$, the state space of the resource state variable, $\mathcal{R}_{t}$, is being initialised for all $t \in \mathcal{T}$ through simple "exploration" to find all feasible resource states subject to $\mathcal{A}_{S_{t}}$, and by using the resource transition function $S^{R}(\cdot)$ to step forward in time. On the other hand, the state space of the information state variable, $\mathcal{I}_{t}$, is generated by simulation (Monte Carlo sampling) while applying the information transition function $S^{I}(\cdot)$, resulting in a set of $|\Omega|$ independent sample realisations $\left\{I_{t}(\omega): \omega \in \Omega\right\}$ for all $t \in \mathcal{T}$. While the latter part of the above procedure is standard in the literature (e.g. see Glasserman (2003)), the former is a direct necessity of our portfolio approach yet uncommon in the real options literature, in which resource states are either not modelled explicitly or scalar. 
Using the initialisations of $\mathcal{R}_{t}$ and $\mathcal{I}_{t}$ for all $t \in \mathcal{T}$ from the forward pass, the backward induction (or pass) determines an approximate value of the problem (2)-(5). Starting at $\max \mathcal{T}$ and moving backwards to $\min \{\mathcal{T} \backslash 0\}$, for each resource state $R_{t} \in\left\{R_{t}^{\prime} \in\right.$ $\left.\mathcal{R}_{t}: b^{D}\left(N_{t}^{\prime}\right) \neq \emptyset\right\}$ the following three steps are performed: (i) applying least-squares regression, determine the optimal values of the coefficients $\left(\alpha_{l}\left(S^{R}\left(R_{t}, a_{t}\right)\right)\right)_{l=0}^{L}$ for all $a_{t} \in \mathcal{A}_{S_{t}}{ }^{9}$,

$$
\left(\hat{\alpha}_{l}\left(R_{t+\Delta_{h}}\right)\right)_{l=0}^{L}=\underset{\left(\alpha_{l}(\cdot)\right)_{l=0}^{L}}{\arg \min }\left\{\sum_{\omega \in \Omega}\left[e^{-k \Delta_{h}} \bar{G}_{t+\Delta_{h}}\left(S_{t+\Delta_{h}}(\omega)\right)-\sum_{l=0}^{L} \alpha_{l}\left(R_{t+\Delta_{h}}\right) \phi_{l}\left(I_{t}(\omega)\right)\right]^{2}\right\},
$$

where $R_{t+\Delta_{h}}=S^{R}\left(R_{t}, a_{t}\right)$ and $S_{t+\Delta_{h}}(\omega)=\left(R_{t+\Delta_{h}}, I_{t+\Delta_{h}}(\omega)\right)$; (ii) using the result of (i) with $\hat{\Phi}_{t}^{L}\left(S_{t}, a_{t}\right)$ as in (6), compute the pathwise optimisers $\hat{a}_{t}(\omega)$ of the pathwise approximation of the problem (2)-(5) for all $\omega \in \Omega$ :

$$
\begin{aligned}
\hat{a}_{t}(\omega)=\underset{a_{t}(\omega)}{\arg \max }\left\{\Pi_{t}\left(S_{t}(\omega), a_{t}(\omega)\right)+\max \left\{\check{\Phi}_{t}\left(R_{t}, a_{t}(\omega)\right), \hat{\Phi}_{t}^{L}\left(S_{t}(\omega), a_{t}(\omega)\right)\right\}\right\} \\
\text { s.t. } \quad a_{t}(\omega) \in \mathcal{A}_{S_{t}(\omega)}, \\
\quad a_{t h}(\omega) \in\{0,1\}, \quad \forall h \in b^{D}\left(N_{t}\right),
\end{aligned}
$$

where $\check{\Phi}_{t}\left(R_{t}, a_{t}(\omega)\right)$ is a lower bound on the continuation value, given $R_{t}$ and $a_{t}(\omega)$; (iii) using the result of (ii), approximate the optimal portfolio value $G_{t}\left(S_{t}\right)$ given $S_{t}$ at time $t$ along each path $\omega \in \Omega$ by:

$$
\bar{G}_{t}\left(S_{t}(\omega)\right)=\Pi_{t}\left(S_{t}(\omega), \hat{a}_{t}(\omega)\right)+e^{-k \Delta_{h}} \bar{G}_{t+\Delta_{h}}\left(S^{R}\left(R_{t}, \hat{a}_{t}(\omega)\right), I_{t+\Delta_{h}}(\omega)\right)
$$

At time 0 , however, these three steps cannot by applied as $S_{0}=S_{0}(\omega)$, for all $\omega \in \Omega$, but now the conditional expectation in (2) can be computed directly by taking the average over all $|\Omega|$ pathwise continuation values $\bar{G}_{\Delta_{h}}\left(S^{R}\left(R_{0}, a_{0}\right), I_{\Delta_{h}}(\omega)\right)$, so the approximate value of the portfolio of interdependent real options is then:

$$
\bar{G}_{0}\left(S_{0}\right)=\max _{a_{0} \in \mathcal{A}_{S_{0}}}\left(\Pi_{0}\left(S_{0}, a_{0}\right)+\frac{1}{|\Omega|} \sum_{\omega \in \Omega} e^{-k \Delta_{h}} \bar{G}_{\Delta_{h}}\left(S^{R}\left(R_{0}, a_{0}\right), I_{\Delta_{h}}(\omega)\right)\right)
$$

where $a_{0, h} \in\{0,1\}$, for all $h \in b^{D}\left(N_{0}\right)$.

In the first and third step above as well as in 12 , the pathwise (approximated)

\footnotetext{
${ }^{9}$ To simplify the presentation of the algorithm, the special case of transitions leading to terminal nodes with deterministic values (no approximation needed) is not treated separately here.
} 
continuation values $\bar{G}_{t+\Delta_{h}}(\cdot)$ and $\bar{G}_{\Delta_{h}}(\cdot)$ are already known at times $t$ and 0 , respectively, since these are defined recursively. Importantly, unlike Tsitsiklis and Van Roy (2001), we use $\hat{\Phi}_{t}^{L}(\cdot)$ only for the sake of computing the pathwise optimal decisions in step (ii), but the actually realised, pathwise continuation values, $\bar{G}_{t+\Delta_{h}}(\cdot)$, to approximate $G_{t}(\cdot)$ by $\bar{G}_{t}(\cdot)$ in step (iii), which is in accordance with the approach of Longstaff and Schwartz (2001) and results in a comparatively smaller absolute bias as well as less and much slower accumulating approximation errors, as recently demonstrated by Stentoft (2014). In addition, assuming a deterministic lower bound exists, we correct obviously erroneous approximations of the continuation value by replacing $\hat{\Phi}_{t}^{L}(\cdot)$ in $(8)$ with $\max \left\{\check{\Phi}_{t}(\cdot), \hat{\Phi}_{t}^{L}(\cdot)\right\}$; e.g., $\check{\Phi}_{t}=0$ for an American option, as in Glasserman (2003). A summary of the backward induction procedure is shown by Algorithm 1 .

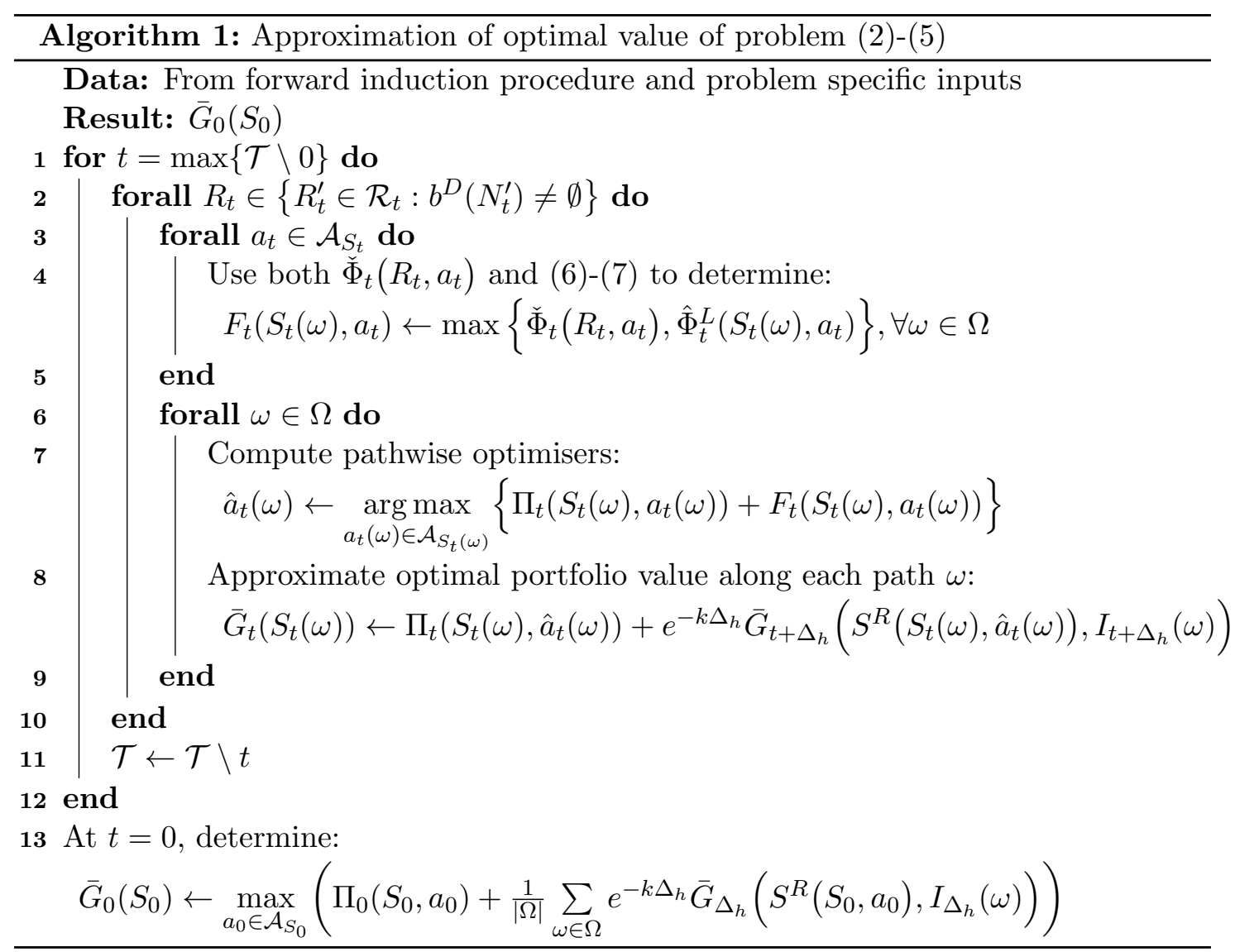

Although backward induction procedures are widely applied, the one described above contains several important features that allow us to approximate the value of a portfolio of interdependent real options. Firstly, the backward induction has to be applied for each 
resource state $R_{t} \in \mathcal{R}_{t}$ that does not correspond with a terminal node (no decision needed there), which is a direct consequence of our portfolio approach. By contrast, this is generally not needed for the regression-based pricing of single options with early-exercise features, where $R_{t}$ is commonly not modelled explicitly. Indeed, the pricing algorithms of Tsitsiklis and Van Roy (2001); Longstaff and Schwartz (2001) for American-style options, for example, solely loop over all exercise times $t \in \mathcal{T}$ - i.e. there is no inner loop over $R_{t}-$, whilst assuming that the option has not been exercised prior to $t$. Secondly, two of the procedure's three nested loops perform particular portfolio-related tasks.

In step (i), the optimal coefficients $\left(\hat{\alpha}_{l}\left(S^{R}\left(R_{t}, a_{t}\right)\right)\right)_{l=0}^{L}$ are determined for every feasible decision $a_{t}$, given $R_{t}$, which consequently satisfy the set of constraints describing the interdependencies between real options in the portfolio. But this is generally not necessary for the pricing of single, well-defined options, which often feature trivial decision spaces; for instance, consider the simple "hold vs. exercise" decision underlying an American-type option shown in Figure A.3. Also, unlike the approach of Longstaff and Schwartz (2001), we include in the regression (7) all $|\Omega|$ paths thereby improving approximation accuracy (Areal et al., 2008: Stentoft, 2014). It is important to note that doing so is in fact necessary here as the exercise decision is required for all paths (and not just for in-the-money-paths as in Longstaff and Schwartz (2001)) given that the pathwise values of $\Pi_{t}(\cdot)$ generally differ for compound real options within the portfolio.

In step (ii), the optimal decision $\hat{a}_{t}(\omega)$ along path $\omega$ is computed by optimally solving the integer program (8)- 10$]$ for each path $\omega \in \Omega$, giving $|\Omega|$ pathwise optimisers $\hat{a}_{t}(\omega)$. These represent the decision vectors that maximise the pathwise approximated portfolio value given $S_{t}$ at time $t$ whilst satisfying the constraints in $\mathcal{A}_{S_{t}(\omega)}$. In contrast, the decision-making process underlying most existing regression-based pricing algorithms boils down to simply comparing $\Pi_{t}(\cdot)$ with $\hat{\Phi}_{t}^{L}(\cdot)$, e.g. by $\max \left\{\Pi_{t}(\cdot), \hat{\Phi}_{t}^{L}(\cdot)\right\}$, under total (or complete) enumeration of all mutually exclusive alternatives; e.g., two in the case of Bermudan (Glasserman, 2003) and purchase swing options (Nadarajah et al., 2017), several in the case of switching (Tsekrekos et al., 2012) and storage options (Nadarajah et al. 2017). Hence, these algorithms are limited to problems where the number of possible actions is small enough to enumerate because total enumeration tends to become impractical when the decision is a vector and when there are no appropriate constraints such as "mutual exclusivity". Rather than relying on such a brute force strategy, in our algorithm an integer programming formulation is used to optimise pathwise decisions. 


\subsection{Computational efficiency and numerical accuracy}

Since the IDSR approach uses a continuation function approximation, it shares a number of desirable properties with the LSM approach. For example, convergence results have been provided by Longstaff and Schwartz (2001); Clément et al. (2002); Stentoft (2004b). The robustness of the approach to different choices of basis functions has been shown by Moreno and Navas (2003) and more recently by Tsekrekos et al. (2012), as well as with an emphasis on the tade-off between computational time and precision by Stentoft (2004a). More recently, Areal et al. (2008) have demonstrated various ways to improve the accuracy of the valuation approach by investigating the influence of different regression algorithms, several variance reduction techniques, various polynomial families, as well as varying numbers of both model dimensions $(L)$ and paths $(|\Omega|)$. Furthermore, Longstaff and Schwartz (2001) recommend appropriate scaling before performing the least-squares regression in order to avoid numerical errors and computational underflows.

By its nature, the proposed algorithm applies the same strategy regarding mutually exclusive options as the one of Areal et al. (2008), who has shown that this strategy provides faster and more accurate results than the algorithm presented by Gamba (2003). Despite providing a lower bound on the true value of the portfolio of interdependent real options, the IDSR approach can be extended by applying duality theory (e.g. see (Haugh and Kogan, 2007)) to allow for the estimation of accurate upper (dual) bounds similar to Nadarajah et al. (2017). Furthermore, the integer programming problem (8)-(10) can be solved efficiently by standard solvers applying such algorithms as branch and bound (Ahmed et al., 2003). Lastly, while more advanced regression methods such as nonparametric models (e.g. kernel regression, local averaging, smoothing splines, and neural networks (Carriere, 1996; Judd, 1998; Powell, 2011)) can lead to more accurate results with lower computational efforts (e.g. see Kohler (2010)), they are generally not readily applicable in high-dimensional settings and even low-dimensional problems can be comparatively complex (Pizzi and Pellizzari, 2002, Kohler, 2010; Powell, 2011).

\section{Evaluating complex natural resource investments}

In this section we demonstrate how the IDSR approach can be used to evaluate a complex natural resource investment by first presenting the problem setting, then detailing the modelling of the problem, and lastly approximating the value of the investment.

\subsection{Problem setting}

The problem considered in this Section is a complex yet important and realistic extension (Savolainen, 2016) of the example of valuing a copper mine, which was proposed 
by Brennan and Schwartz (1985) and solved by the authors using a finite difference method, and which has been used by Sabour and Poulin (2006); Cortazar et al. (2008); Tsekrekos et al. (2012) as a benchmark to assess the LSM approach. The original copper mine example of Brennan and Schwartz has only considered a limited set of options (option to temporarily close the mine and possibly reopen as well as early abandonment option) and only treated the price of copper to be uncertain. Here we substantially extend their example in two respects.

First, in terms of portfolio of real options considered, we integrate the option to defer (or delay) the development of the copper mine as proposed by Gamba (2003) and valued by the author using the LSM approach; however, Sabour and Poulin (2006) discussed the findings of Gamba and showed that there are some inconsistencies related to both numerical results (mine value cyclic in copper price) and switching decisions obtained. In addition to the option to defer, our example takes into account the option to irreversibly expand production capacity of the operating mine, which was proposed by Cortazar and Casassus (1998) and solved by the authors using partial differential equations; however, the authors did not consider the option to abandon the project.

The second extension is in terms of uncertainties considered. We replace the onefactor setting considered in Brennan and Schwartz (stochastic copper price) by applying the three-factor model (copper price, convenience yield and interest rate) of Schwartz (1997), which has been analysed in the context of the original copper mine example by Tsekrekos et al. (2012) using the LSM approach. In addition to these three stochastic factors, we introduce a forth factor by treating the extraction (production) costs of copper to be uncertain, as argued for by Slade (2001). It is important to note that traditional valuation methods (e.g. lattice and finite difference) are impractical for the problem considered here given its large size in terms of portfolio of options and uncertainties.

\subsection{Modelling}

The flexibilities inherent in the mine project are represented by the ID in Figure1. It contains 5 decision nodes and 3 terminal nodes, as well as 18 transitions that link these nodes, resulting in $\mathcal{N}=\{1,2, \ldots, 8\}$ and $\mathcal{H}=\{1,2, \ldots, 18\}$. The duration of transition $h \in \mathcal{H}$ is $\Delta_{h}$ years. When the mine is Undeveloped, the decision maker may decide either to Defer (1) development or Develop (2) the mine, both of which can be done for up to $T_{1}^{\max }$ years, after which the right to develop the mine expires. Once developed and in mode Opened-I, the decision maker has to decide whether to Operate (4) for the duration of $\Delta_{4}$ while extracting an amount $q^{I} \Delta_{4}$ of copper, irreversibly Expand (5) the

mine operation by increasing extraction rate from $q^{I}$ to $q^{I I}$, temporarily Close (6), or 


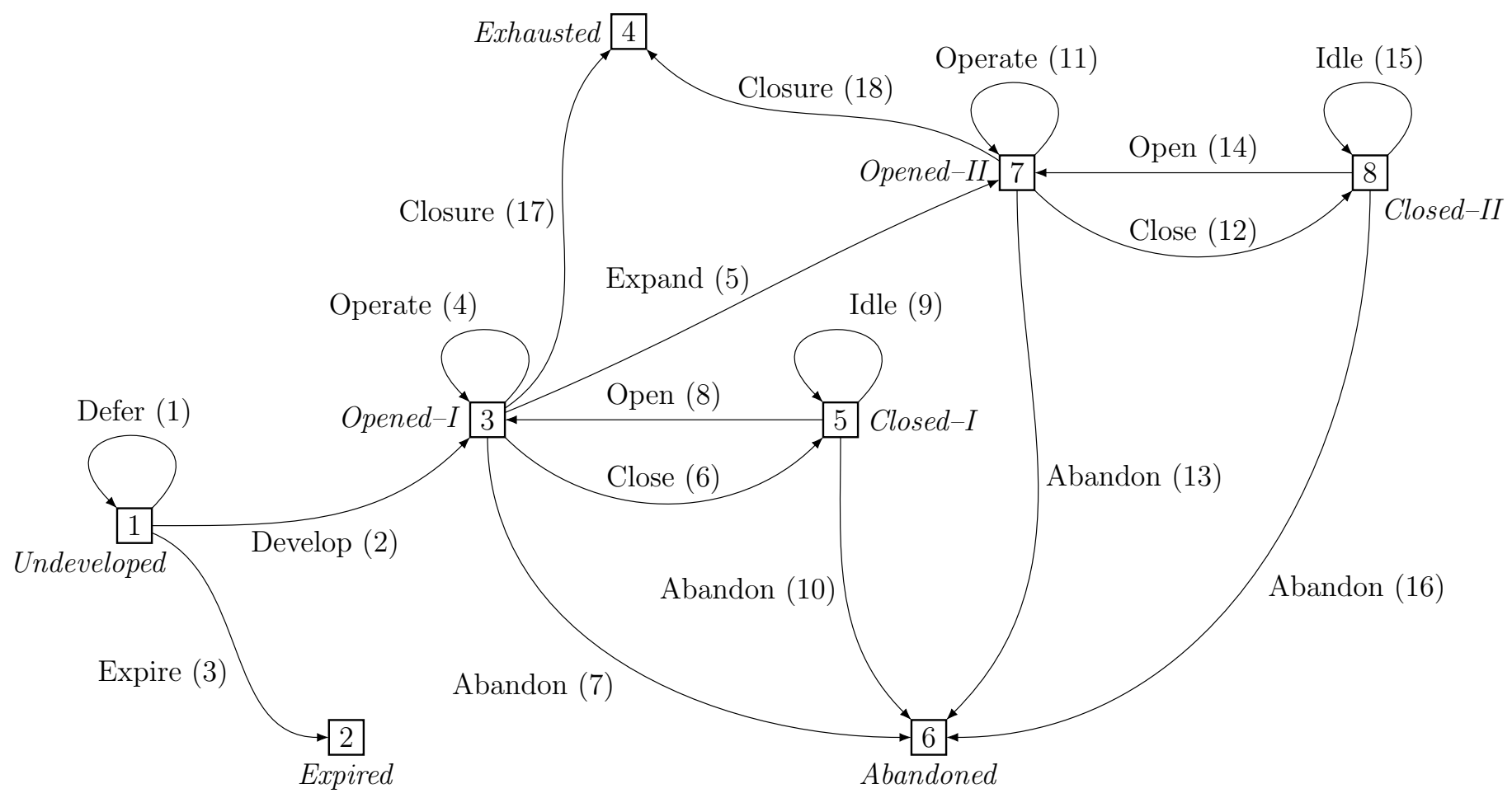

Figure 1: Influence diagram for the mine development project.

irreversibly Abandon (7) the project. On the other hand, if the mine is Closed-I (or Closed-II), the available transitions are to keep the mine Idle (9 or 15), Open (8 or 14) it again, or irreversibly Abandon (10 or 16) the project. In either operating mode, however, the mine closures if the commodity inventory with initial inventory $Q_{0}$ is fully depleted. Also, the project has to be Abandoned when reaching its lifetime of $T_{2}^{\max }$ years.

Let the decision node, the inventory of the mine, and the remaining time to develop the mine/lifetime of the mine at time $t$ be denoted by $N_{t}, Q_{t}$, and $T_{t}$, respectively, as well as let the spot price of copper, the instantaneous convenience yield, the instantaneous risk-free interest rate, and the (per-unit) production cost at time $t$ be denoted by $X_{t}, \delta_{t}$, $r_{t}$, and $A_{t}$, respectively. Thus, the resource and information state component are given by $R_{t}=\left(t, N_{t}, Q_{t}, T_{t}\right)$ and $I_{t}=\left(X_{t}, \delta_{t}, r_{t}, A_{t}\right)$, respectively, hence $S_{t}=\left(R_{t}, I_{t}\right)$.

The binary decision variables related to the transitions available at decision node $N_{t}$, 
$a_{t}=\left(a_{t h}\right)_{h \in b^{D}\left(N_{t}\right)}$, have to satisfy the feasible region $\mathcal{A}_{S_{t}}$, which is defined by:

$$
\begin{cases}\sum_{h \in b^{D}\left(N_{t}\right)} a_{t h}=1, & \forall N_{t} \in \mathcal{N} \backslash\{2,4,6\}, \\ a_{t 1} T_{1}^{\text {max }}<T_{1}^{\text {max }}+T_{t}, & \\ a_{t 3} T_{t}=0, & \\ a_{t h} q^{I} \Delta_{h} \leq Q_{t}, & \forall h \in\{4,8\}, \\ a_{t h} q^{I I} \Delta_{h} \leq Q_{t}, & \forall h \in\{11,14\}, \\ a_{t h_{1}}+a_{t h_{2}}+T_{t}>0, & \forall\left(h_{1}, h_{2}\right) \in\{(7,17),(13,18)\}, \\ a_{t h}+T_{t}>0, & \forall h \in\{10,16\}, \\ a_{t h}+Q_{t}>0, & \forall h \in\{17,18\}, \\ a_{t h} Q_{t}=0, & \forall h \in\{17,18\},\end{cases}
$$

where $a_{t h} \in\{0,1\}, \forall h \in \mathcal{H}$, and the set of outgoing transitions of node $N_{t}$ is:

$$
b^{D}\left(N_{t}\right)= \begin{cases}\{1,2,3\}, & \text { if } N_{t}=1, \\ \{4,5,6,7,17\}, & \text { if } N_{t}=3, \\ \{11,12,13\}, & \text { if } N_{t}=5, \\ \{14,15,16\}, & \text { if } N_{t}=7, \\ \{\}, & \text { if } N_{t}=8, \\ & \text { otherwise }\end{cases}
$$

These constraints accomplish the following: constraints (13) make sure that exactly one transition is made at a decision node; constraints (14) and (15) ensure the development can only be delayed if $T_{t}>0$ and the right to develop can only expire at $T_{t}=0$, respectively; constraints (16) and (17) make sure the inventory does not become negative; constraints (18), 20) and (21) require the opened mine to closure if and only if $Q_{t}=0$, and to be abandoned if both $T_{t}=0$ and $Q_{t}>0$; and, finally, constraints 119 make sure the mine is abandoned if closed at $T_{t}=0$. It is important to note that constraints (14) and (18)- 20 can be easily transformed into (weak) inequality constraints if needed.

Subsequently, the resource state $R_{t}$ evolves deterministically to $R_{t+\Delta_{h}}$, with the transition of $t$ being rather trivial as it simply evolves from $t$ to $t+\Delta_{h}$ after having made transition $h$. The evolution of $N_{t}$ is implicitly described by the adjacency matrix of the digraph $(\mathcal{N}, \mathcal{H})$, which is not shown here for brevity. The evolution of $Q_{t}$ and $T_{t}$ 
are specified by the following transition equations for all $h \in \mathcal{H}$ :

$$
\begin{aligned}
Q_{t+\Delta_{h}} & =Q_{t}-q^{I} \Delta_{h}\left(a_{t 4}+a_{t 8}\right)-q^{I I} \Delta_{h}\left(a_{t 11}+a_{t 14}\right), \\
T_{t+\Delta_{h}} & = \begin{cases}T_{2}^{\max }-\left(T_{1}^{\max }-T_{t}\right)-\Delta_{2}, & \text { if } a_{t 2}=1, \\
\max \left(T_{t}-\Delta_{h}, 0\right), & \text { otherwise, }\end{cases}
\end{aligned}
$$

where $T_{0}=T_{1}^{\max }$.

On the other hand, the information state $I_{t}$ evolves stochastically to $I_{t+\Delta_{h}}$ under the risk-neutral measure according to the following discrete diffusion processes. As reported by Tsekrekos et al. (2012), the discretised version of the joint stochastic process for the three factors of Schwartz (1997) is given by:

$$
\begin{gathered}
X_{t+\Delta_{h}}=X_{t} \exp \left\{\left(r_{t}-\delta_{t}-\frac{\sigma_{x}^{2}}{2}\right) \Delta_{h}+\sigma_{x} \sqrt{\Delta_{h}} \epsilon_{t+\Delta_{h}}^{x}\right\}, \\
\delta_{t+\Delta_{h}}=\left(1-e^{-\kappa_{\delta} \Delta_{h}}\right) \theta_{\delta}+e^{-\kappa_{\delta} \Delta_{h}} \delta_{t}+\sigma_{\delta} \sqrt{\frac{1-e^{-2 \kappa_{\delta} \Delta_{h}}}{2 \kappa_{\delta}}} \epsilon_{t+\Delta_{h}}^{\delta}, \\
r_{t+\Delta_{h}}=\left(1-e^{-\kappa_{r} \Delta_{h}}\right) \theta_{r}+e^{-\kappa_{r} \Delta_{h}} r_{t}+\sigma_{r} \sqrt{\frac{1-e^{-2 \kappa_{r} \Delta_{h}}}{2 \kappa_{r}}} \epsilon_{t+\Delta_{h}}^{r},
\end{gathered}
$$

where $\sigma_{x}, \sigma_{\delta}$ and $\sigma_{r}$ are the standard deviations of changes in $X_{t}, \delta_{t}$ and $r_{t}$, respectively; $\kappa_{\delta}$ and $\kappa_{r}$ are positive mean reversion (speed of adjustment) coefficients; $\theta_{\delta}$ and $\theta_{r}$ are the long run mean of convenience yield and interest rate, respectively; and $\epsilon_{t+\Delta_{h}}^{x}$, $\epsilon_{t+\Delta_{h}}^{\delta}$ and $\epsilon_{t+\Delta_{h}}^{r}$ are correlated standard normal random variables (mean 0, variance 1) with correlation coefficients $\rho_{x, \delta}, \rho_{x, r}$ and $\rho_{\delta, r}$ (the correlation matrix equals the covariance matrix $\Sigma$ here, see Glasserman (2003)). For the evolution of $A_{t}$, as suggested by Slade (2001), we consider a mean-reverting process, in particular we use the following approximation of the geometric mean reversion described by Metcalf and Hassett (1995):

$$
A_{t+\Delta_{h}}=\left(A_{0} e^{\pi t}-A_{t}\right) \kappa_{a} A_{t} \Delta_{h}+e^{\pi \Delta_{h}} A_{t}+\sigma_{a} A_{t} \sqrt{\Delta_{h}} \epsilon_{t+\Delta_{h}}^{a},
$$

where $\pi$ is the cost inflation rate, $\kappa_{a}$ is a positive mean reversion coefficient, $\sigma_{a}$ is the standard deviation of the production cost, and $\epsilon_{t+\Delta_{h}}^{a}$ is a standard normal random variable (mean 0 , variance 1 ), which is assumed to be uncorrelated with the ones above and whose increments are independently and identically distributed. 
The deterministic payoff obtained at time $t$ when making transition $h$ given $I_{t}$ is:

$$
\begin{aligned}
\Pi_{t}\left(I_{t}, a_{t}\right)= & -I_{t}^{d} a_{t 2}+\left[q^{I}\left(X_{t}-A_{t}\right)-f^{I}\left(X_{t}, A_{t}\right)\right] \Delta_{h}\left(a_{t 4}+a_{t 8}\right)-K_{t}^{c, I} a_{t 6}-K_{t}^{o, I} a_{t 8} \\
& -M_{t}^{I} \Delta_{h}\left(a_{t 6}+a_{t 9}\right)-I_{t}^{e} a_{t 5}+\left[q^{I I}\left(X_{t}-A_{t}\right)-f^{I I}\left(X_{t}, A_{t}\right)\right] \Delta_{h}\left(a_{t 11}+a_{t 14}\right) \\
& -K_{t}^{c, I I} a_{t 12}-K_{t}^{o, I I} a_{t 14}-M_{t}^{I I} \Delta_{h}\left(a_{t 12}+a_{t 15}\right),
\end{aligned}
$$

where $I_{t}^{d}=I_{0}^{d} e^{\pi t}$ is the development cost at time $t, f^{I}\left(X_{t}, A_{t}\right)=\tau_{1} q^{I} X_{t}+\max \left\{\tau_{2} q^{I}\left(X_{t}(1-\right.\right.$ $\left.\left.\left.\tau_{1}\right)-A_{t}\right), 0\right\}$ is the sum of royalties and income tax paid at time $t$ with $\tau_{1}$ the royalty rate and $\tau_{2}$ the income tax rate; $M_{t}^{I}=M_{0}^{I} e^{\pi t}$ is the maintenance cost at time $t$; $K_{t}^{c, I}=K_{0}^{c, I} e^{\pi t}$ and $K_{t}^{o, I}=K_{0}^{o, I} e^{\pi t}$ are the costs to switch to the Closed-I and Opened$I$ node at time $t$, respectively; and $I_{t}^{e}=I_{0}^{e} e^{\pi t}$ is the expansion cost at time $t$. For costs/revenues related to the Closed-II and Opened-II nodes simply replace "I" with "II" in the above definitions. For the sake of simplicity, if $Q_{t}<q^{I I}$ then the payoff associated with transitions 11 and 14 equals the one of transitions 4 and 8, respectively.

\subsection{Valuation}

For valuation, we used the parameter values of Brennan and Schwartz (1985) for the copper mine and of Tsekrekos et al. (2012) for the three-factor model. The initial development cost and the initial expansion cost are estimated at US $\$ 8$ millions $\left(I_{0}^{d}\right)$ and US $\$ 4$ millions $\left(I_{0}^{e}\right)$, respectively. In addition, we consider the following: the possibility to defer development for up to two years (i.e. $\left.T_{1}^{\max }=2\right)$; a lifetime of 45 years $\left(T_{2}^{\max }\right)$; no values associated with terminal nodes (i.e. $G_{t}^{T}\left(S_{t}\right)=0$, for all $S_{t} \in\left\{S_{t}^{\prime} \in \mathcal{S}_{t}: b^{D}\left(N_{t}^{\prime}\right)=\emptyset\right\}$ ); and 5 decisions to be made per year (i.e. $\Delta_{h}=1 / 5$, for $h \in \mathcal{H} \backslash\{3,7,10,13,16,17,18\}$, and 0 otherwise). Also, we considered 100,000 $(|\Omega|)$ sample paths (half of which antithetic for variance reduction) and complete sets of the first five (i.e. $L=4$ ) Legendre/Hermite polynomials, as well as applied a singular value decomposition (SVD) algorithm with properly scaled basis functions to avoid numerical problems when solving the leastsquares regression in (7). For an analysis of the effects of different parametric models see Appendix C. The chosen input data for this example are summarised in Table 1.

The forward induction procedure for this problem consists of the following steps:

1. Determine the set of decision times, $\mathcal{T}_{n}$, for all decisions nodes $n \in\{1,3,5,7,8\}$, 
Table 1: Input data for hypothetical copper mine adapted from Brennan and Schwartz (1985); Tsekrekos et al. (2012) and own estimates.

\begin{tabular}{|c|c|c|c|}
\hline Description & Parameter & Value & Unit \\
\hline \multicolumn{4}{|l|}{ Mine } \\
\hline Output rate & $q^{I}\left(q^{I I}\right)$ & $10(20)$ & Mlbs/year \\
\hline Initial inventory & $Q_{0}$ & 150 & Mlbs \\
\hline Initial cost of opening & $K_{0}^{o, I}\left(K_{0}^{o, I I}\right)$ & $0.20(0.40)$ & $\$ \mathrm{~m}$ \\
\hline Initial cost of closing & $K_{0}^{c, I}\left(K_{0}^{c, I I}\right)$ & $0.20(0.40)$ & $\$ \mathrm{~m}$ \\
\hline Initial maintenance cost & $M_{0}^{I}\left(M_{0}^{I I}\right)$ & $0.50(1.00)$ & $\$ \mathrm{~m} /$ year \\
\hline Cost inflation rate & $\pi$ & $8 \%$ & year $^{-1}$ \\
\hline Initial development cost & $I_{0}^{d}$ & 8 & $\$ \mathrm{~m}$ \\
\hline Initial expansion cost & $I_{0}^{e}$ & 4 & $\$ \mathrm{~m}$ \\
\hline Expiration of development right & $T_{1}^{\max }$ & 2 & year \\
\hline Lifetime of copper mine project & $T_{2}^{\max }$ & 45 & year \\
\hline \multicolumn{4}{|l|}{ Production cost } \\
\hline Initial average production cost & $A_{0}$ & 0.50 & $\$ / \mathrm{lbs}$ \\
\hline Speed of mean reversion in production cost & $\kappa_{a}$ & 0.20 & - \\
\hline Standard deviation of production cost & $\sigma_{a}$ & $15 \%$ & year $^{-1}$ \\
\hline \multicolumn{4}{|l|}{ Copper $^{\mathrm{a}}$} \\
\hline Price variance & $\sigma_{x}^{2}$ & $8 \%$ & year $^{-1}$ \\
\hline Initial convenience yield & $\delta_{0}^{\infty}$ & $1 \%$ & year $^{-1}$ \\
\hline Speed of mean reversion in convenience yield & $\kappa_{\delta}$ & 0.30 & - \\
\hline Long-run mean convenience yield level & $\theta_{\delta}$ & $1 \%$ & year $^{-1}$ \\
\hline Standard deviation of convenience yield & $\sigma_{\delta}$ & $5 \%$ & year $^{-1}$ \\
\hline Initial short-term interest rate & $r_{0}$ & $10 \%$ & year $^{-1}$ \\
\hline Speed of mean reversion in interest rate & $\kappa_{r}$ & 0.50 & - \\
\hline Long-run mean interest rate level & $\theta_{r}$ & $10 \%$ & year $^{-1}$ \\
\hline Standard deviation of interest rate & $\sigma_{r}$ & $1.5 \%$ & year $^{-1}$ \\
\hline \multicolumn{4}{|l|}{ Taxes } \\
\hline Royalty & $\tau_{1}$ & $0 \%$ & - \\
\hline Income & $\tau_{2}$ & $50 \%$ & - \\
\hline Property, Opened/Closed & $\lambda_{1}$ & $2 \%$ & year $^{-1}$ \\
\hline Property, Abandoned & $\lambda_{3}$ & $0 \%$ & year $^{-1}$ \\
\hline
\end{tabular}

${ }^{\mathrm{a}}$ The values of the correlation coefficients are: $\rho_{x, r}=0.15, \rho_{x, \delta}=0.40$, and $\rho_{\delta, r}=0.10$.

${ }^{\mathrm{b}}$ The value of the discount rate at time $t, k_{t}$, is $r_{t}+\lambda_{1}$.

forming subsets of $\mathcal{T}$ :

$$
\mathcal{T}_{n}= \begin{cases}\left\{i \Delta_{1}: i \in \mathbb{Z}_{\geq 0}, 0 \leq i \Delta_{1} \leq T_{1}^{\text {max }}\right\}, & \text { if } n=1, \\ \left\{i \Delta_{1}: i \in \mathbb{Z}_{\geq 0}, \tau_{1} \in \mathcal{T}_{1}, \tau_{1}+\Delta_{1} \leq i \Delta_{1} \leq T_{2}^{\text {max }},\right. & \text { if } n=3, \\ \left\{i \Delta_{1}: i \in \mathbb{Z}_{\geq 0}, \tau_{1} \in \mathcal{T}_{1}, \tau_{1}+2 \Delta_{1} \leq i \Delta_{1} \leq T_{2}^{\text {max }},\right. & \text { if } n \in\{5,7\} \\ \left\{i \Delta_{1}: i \in \mathbb{Z}_{\geq 0}, \tau_{1} \in \mathcal{T}_{1}, \tau_{1}+2 \Delta_{1} \leq i \Delta_{1} \leq T_{2}^{\text {max }},\right. & \text { if } n=8,\end{cases}
$$


2. Use (27), 26) and 25] to sample $|\Omega|$ paths of $r_{t}, \delta_{t}$ and $X_{t}$, respectively, giving $\left(X_{t}(\omega), \delta_{t}(\omega), r_{t}(\omega)\right)_{\omega \in \Omega}, \forall t \in \mathcal{T}$

3. Use $(28)$ to sample $|\Omega|$ paths of $A_{t}$, giving $\left(A_{t}(\omega)\right)_{\omega \in \Omega}, \forall t \in \mathcal{T}$

4. Generate the possible resource state space $\mathcal{R}_{t}$ for each decision node and time

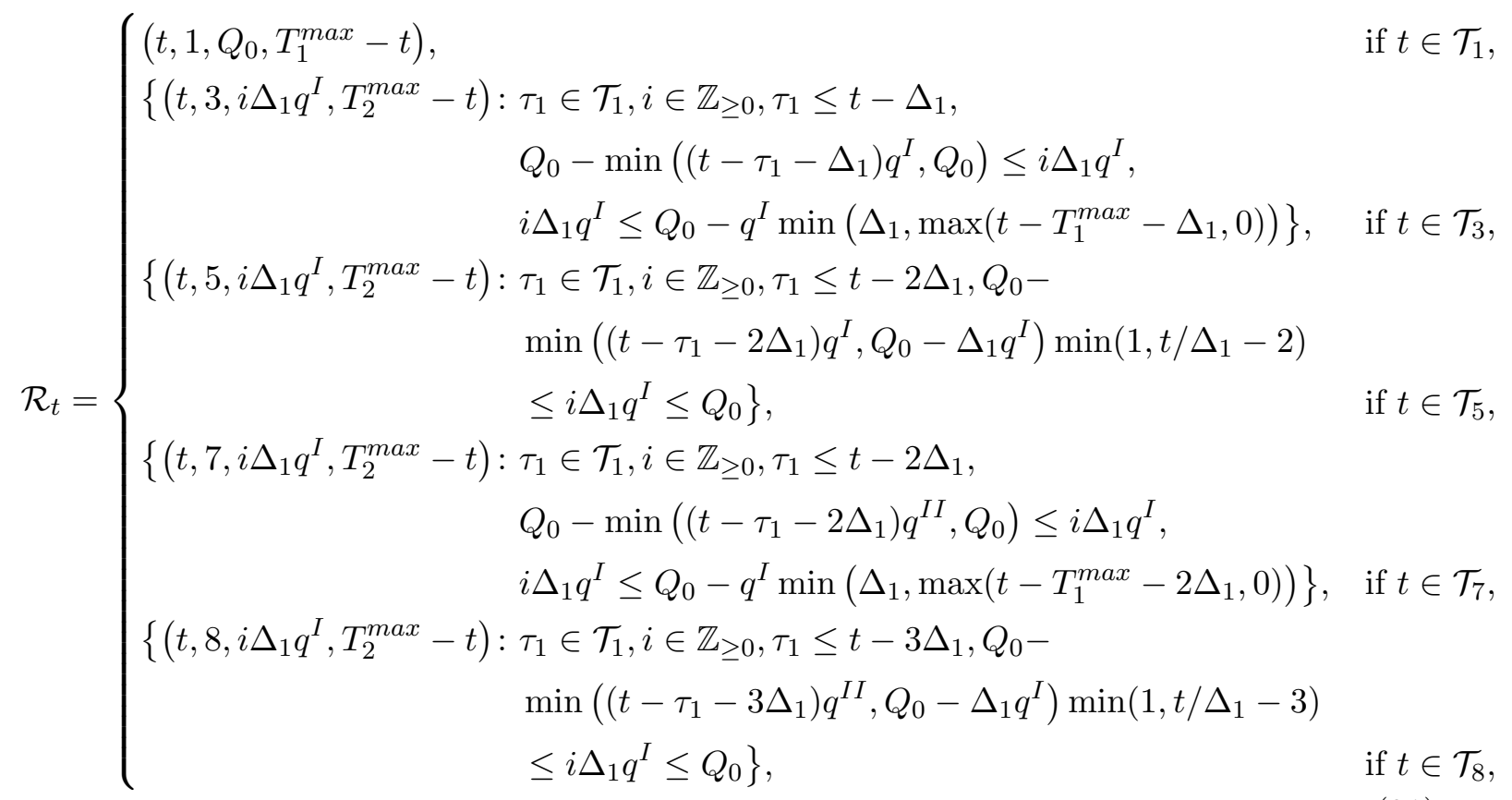

The valuation algorithm was implemented in MATLAB.

\section{Results and discussion}

This section begins with an analysis of the way in which the mine value with different configurations of option portfolios is affected by the initial copper price, $X_{0}$. Table 2 summarises the results when $X_{0}$ is in the range from $\$ 0.30$ to 1.00 per pound. Columns $(\dagger)$ and $(\ddagger)$ give the expected values of the mine under "now-or-never strategies", which assume it must be either (developed and in case $(\ddagger)$ expanded immediately and then) operated at the rate of 10 and 20 Mlbs/year, respectively, until the inventory is fully exhausted, or left undeveloped. As can be seen, the value of the mine with fixed-outputrate $q^{I}\left(q^{I I}\right)$ is positive for copper prices of $\$ 0.70(0.80)$ per pound and above, making development of the mine viable, but only for $X_{0}=1.00$ is it optimal to have an expanded mine with fixed-output-rate $q^{I I}$. While these price levels are not the critical prices (i.e.

\footnotetext{
${ }^{10}$ For simplicity, we assume $Q_{t} \bmod \left(q^{I} \Delta_{1}\right)=0$.
} 


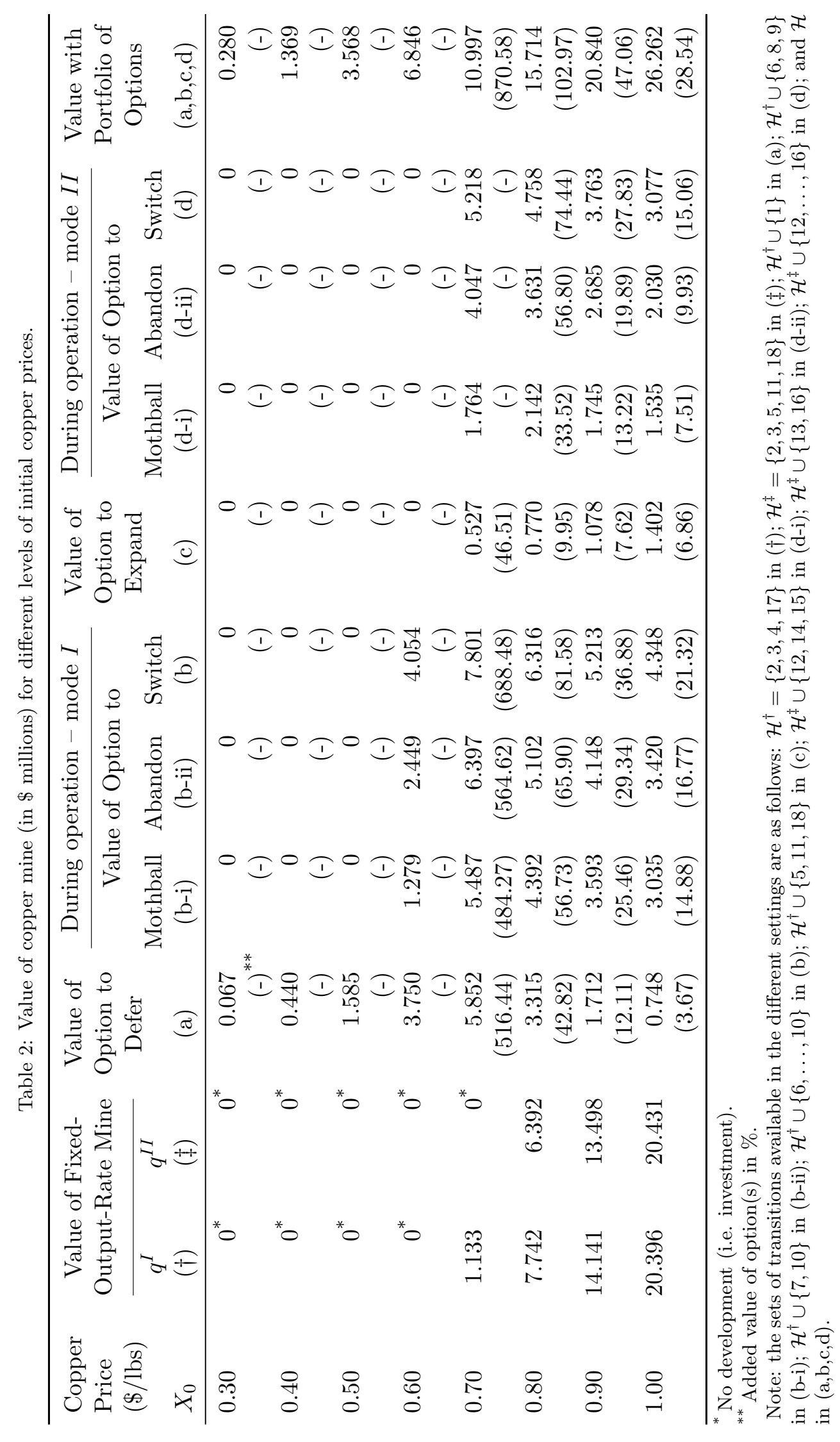


the point at which it becomes optimal to invest, which largely depends on the input data of Table 1), these can be estimated simply and accurately through numerical simulation.

As can be seen from Table 2, the flexibility provided by individual real options can add considerable value. Column (a) displays the value added to the mine with fixedoutput-rate $q^{I}$ when development can be deferred for up to 2 years. Determined by the difference between the value of the fixed-output-rate mine with the option to defer (not shown here) and column $(\dagger)$, the value of the option to defer is positive for all copper prices listed. This means it adds value in every situation. Its adds sufficient value even when $X_{0} \leq 0.60$, enabling the mine to become economically viable by allowing the mine's development to be deferred. For $X_{0} \leq 0.60$, the value of this option would be much higher if we had used the actual NPV (which is highly negative in these situations) as a benchmark instead of the non-negative value of the fixed-output-rate mine. As expected, the value of this option decreases as $X_{0}$ increases because the ability to defer development is economically less attractive when prices are high.

Considering a developed but not expanded mine, columns (b-i), (b-ii) an (b) report the value of the option to temporarily mothball the operation, to abandon the project during operation and to switch, respectively. These values were determined such that the mine values with these individual options were measured against the value of the mine with fixed-output-rate $q^{I}$ as the benchmark. Having either of these individual options is valuable for copper prices of $\$ 0.60 / \mathrm{lbs}$ and above, showing the mine to be viable in situations where the fixed-output-rate mine does not. At the same time, abandoning the project was found to be more valuable than mothballing, and switching more valuable than abandoning. In fact, representing the portfolio of the option to mothball and to abandon, the option to switch will always be at least as valuable as its constituent options. Although the values of these three options decline as the price increases, their levels remain comparatively high and they decline less strongly than the value of the option to defer. This indicates that operational flexibility is more beneficial than the flexibility associated with investment timing when price levels are high.

Column (c) reports the value of the option to expand, which enables the mine to double its fixed-output-rate to $20 \mathrm{Mlbs} /$ year at any point during operation. Its value is given by the difference between the value of the mine with the option to expand and the maximum of the values shown in columns $(\dagger)$ and $(\ddagger)$. Considering a developed and immediately expanded mine, columns (d-i), (d-ii) an (d) give the value of the option to temporarily mothball the operation, the value of the option to abandon the project during operation and the value of the option to switch, respectively. These values were determined in a similar way to those shown in columns (b-i), (b-ii) an (b), but now 
measured against the value of the mine with fixed-output-rate $q^{I I}$. Comparing the values of columns (d-i), (d-ii) an (d) with the ones of columns (b-i), (b-ii) an (b) shows that mothballing, abandoning and switching during operation of the developed but not expanded mine is more valuable. This implies that operational flexibility to deviate from the extraction of copper is less beneficial if the mine is expanded.

The value of the mine with the portfolio of interdependent real options is shown in column (a,b,c,d). As seen in Table 2 in most cases its value is considerably larger than the value of the mine without options or with only an individual real option. This highlights the substantial added value achieved by considering such a complex portfolio. While the value of the mine with the portfolio increases in $X_{0}$, the absolute difference between this value and the value of the best-performing fixed-output-rate mine decreases as $X_{0}$ increases. This is because flexibility to deviate from the static now-or-never strategy becomes less valuable. However, the relative difference is still over $28 \%$ for the highest copper price considered. Comparing the values of the individual options of columns (a) to (d) shows that the option to expand is the only option whose value increases in the copper price, all other options diminish in value. These results, which are in line with the real options literature, demonstrate the ability of the option to expand to exploit upside potential, and the ability of the other options (i.e. to defer, to mothball, to abandon, and to switch) to limit downside risk when operating margins are lower.

To illustrate the effects of the degrees of different uncertainties on the value of the copper mine, Figure 2 shows for $X_{0}=0.70$ the way in which the standard deviations of the production cost, $\sigma_{a}$, and the copper price, $\sigma_{x}$, effect the investment value. As we would expect, the value of the mine without options, which applies the best-performing static now-or-never strategy (always with fixed-output-rate $q^{I}$ here) and hence does not consider any flexibility, decreases as price uncertainty increases and eventually reaches zero at $\sigma_{x}=0.30\left(\sigma_{x}=0.35\right.$ for $0.09 \leq \sigma_{a} \leq 0.24, \sigma_{x}=0.40$ for $\left.\sigma_{a} \geq 0.27\right)$. On the other hand, the (expected) value of the mine without options, if positive, slightly increases in $\sigma_{a}$ because, although production cost uncertainty increases, average production cost slightly decreases due to the characteristics/parameters of the stochastic process in (28).

Taking into account the portfolio of real options and thus allowing the decision-maker to exploit flexibilities adds substantial value for all degrees of uncertainties considered, especially for high degrees of uncertainties ${ }^{11}$. Increasing $\sigma_{a}$ from 0 to 0.30 generally

\footnotetext{
${ }^{11}$ Note that at $\sigma_{x}=0, X_{t}$ still evolves stochastically due to its dependence on the stochastic factors $\delta_{t}$ and $r_{t}$ in the three-factor model, with the value added by the options portfolio at $\sigma_{a}=\sigma_{x}=0$ amounting to almost $46 \%$ of the value of the project without options.
} 


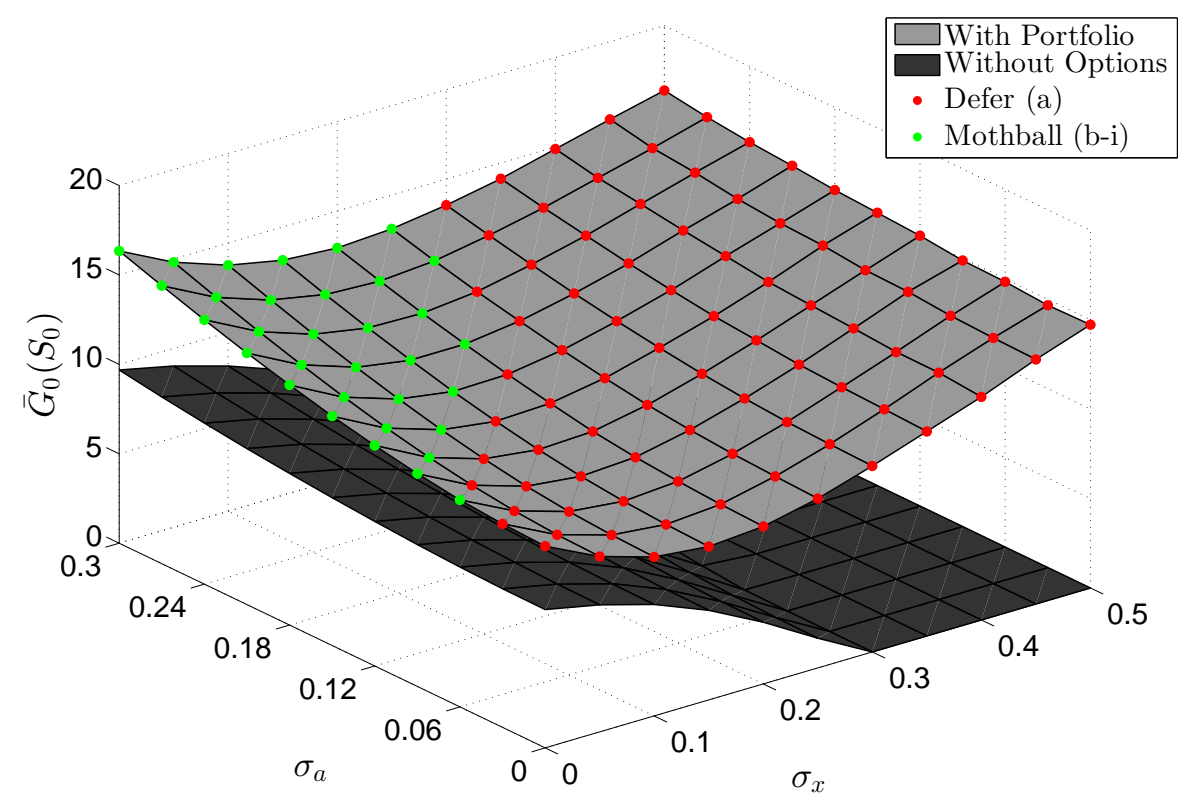

Figure 2: Value of investment project, $\bar{G}_{0}\left(S_{0}\right)$ (in $\$$ millions), with portfolio of real options and without options as well as portfolio's most valuable individual option (filled circles), as a function of degrees of production cost $\left(\sigma_{a}\right)$ and copper price $\left(\sigma_{x}\right)$ uncertainty.

results in appreciably higher values of the mine with the portfolio and this effect tends to be stronger for lower $\sigma_{x}$. In contrast, there is an unexpected twofold effect of the degree of price uncertainty on the value of the mine with options portfolio: values actually decrease in $\sigma_{x}$ for low $\sigma_{x}$-values, but, beginning at a $\sigma_{x}$ of $0.15\left(0.20\right.$ for $\left.\sigma_{a} \geq 0.24\right)$, increase in $\sigma_{x}$; this decrease (increase) tends to be steeper for higher (lower) $\sigma_{a}$-values. The reasons for this somewhat intriguing twofold effect are believed to be the overall volatility in the three-factor model, which depends non-linearly on $\sigma_{x}$ and features a similar pattern to the one shown in Figure 2, as well as portfolio effects which, as mentioned earlier and indicated by the filled circles ${ }^{12}$ in Figure 2, means that the portfolio's individual options are affected differently (beneficially or adversely) by changes of the underlying conditions resulting in positively or negatively affected portfolio values.

\footnotetext{
${ }^{12}$ The portfolio's least valuable options (not shown here) are the option to abandon (b-ii) for very high degrees of uncertainties of $\left(\sigma_{a}, \sigma_{x}\right) \in\{(0.27,0.50),(0.30,0.45),(0.30,0.50)\}$, and either the option to mothball $(\mathrm{d}-\mathrm{i})$ or to abandon (d-ii) for the remainder of degrees of uncertainties.
} 


\section{Conclusions}

This paper presents a new approach for modelling and approximating the value of portfolios of interdependent real options using both influence diagrams and simulationand-regression. Our modelling technique is intuitive and compact; strategic interdependencies between real options are translated into a set of constraints and the risk-neutral dynamics of all underlying uncertainties are directly modelled using (Markovian) stochastic processes. These are then easily implemented in the multi-stage stochastic integer program. To approximate the value of this optimisation problem, we apply simulation and parametric regression and have presented a transparent valuation algorithm. In contrast to existing regression-based valuation algorithms, ours explicitly takes into account vector-valued exercise decisions and the state variable's multidimensional resource component that generally occur in real option portfolios. We demonstrate the ability of this approach to evaluate complex and risky investment projects by evaluating a complex yet realistic natural resource investment that features both a large portfolio of interdependent real options and four stochastic factors. Using this example, we show how our approach can be used to investigate the way in which the value of the portfolio and its individual options are effected by the underlying operating margin and the degrees of different uncertainties. Future work will investigate ways to integrate other types of uncertainties into the modelling framework presented here as well as explore possibilities to improve the efficiency of the simulation-and-regression-based valuation algorithm.

\section{Acknowledgements}

The authors are grateful to the three anonymous reviewers for their valuable comments and suggestions. The authors would like to thank participants at the 19th \& 20th Annual International Real Options Conference, the Annual International Conference on Operations Research (OR2015), the International Symposium for Next Generation Infrastructure, and the INFORMS Annual Meeting $2014 \& 2015$. This work was supported by the Grantham Institute at Imperial College London and the European Institute of Innovation \& Technology's Climate-KIC.

\section{Appendix A. Modelling example of an American option}

The managerial flexibility provided by a standard American-type (Bermudan in discrete-time) option is represented by the ID in Figure A.3. The ID consists of one decision node termed Holding (1) and two terminal nodes, Exercised (2) and Expired 


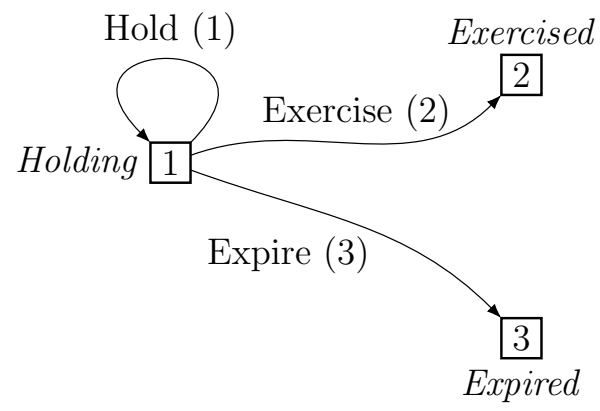

Figure A.3: Influence diagram for American option.

(3), as well as three transitions termed Hold (1), Exercise (2) and Expire (3). Consequently, the sets of nodes and transitions are given by $\mathcal{N}=\{1,2,3\}$ and $\mathcal{H}=\{1,2,3\}$, respectively. When Holding at time $t$, the option holder has to decide whether to exercise the option immediately, or to hold the option until $t+\Delta_{1}$. At the expiration date $T^{\max }$, however, this option automatically expires if not exercised.

\section{Appendix B. Nomenclature}

This appendix contains a summary of most of the notation used in this work.

\begin{tabular}{ll}
\hline & \multicolumn{1}{c}{ Sets and indices } \\
\hline $\mathcal{N}$ & Set of nodes, $\{1, \ldots, N\}$ \\
$\mathcal{H}$ & Set of transitions, $\{1, \ldots, H\}$ \\
$t$ & Time index, $t \in \mathcal{T}$ \\
$\mathcal{T}$ & Set of decision times (or epochs) \\
$\mathcal{S}_{t}$ & State space at time $t$ \\
$\mathcal{R}_{t}$ & Resource state space at time $t$ \\
$\mathcal{I}_{t}$ & Information state space at time $t$ \\
$\omega$ & Sample path, $\omega \in \Omega$ \\
$\Omega$ & Set of sample paths \\
$l$ & Index of summation, $l=0, \ldots, L$, used to specify the $l$-th dimension \\
& of the parametric model, where $l=0$ refers to a constant term \\
\hline & Duration of transition $h \in \mathcal{H}$ \\
\hline$\Delta_{h}$ & Risk-free rate $($ discount factor $)$ \\
$k$ & A basis function (or feature) that extracts information from $I_{t}$ \\
$\phi_{l}\left(I_{t}\right)$ & Dimension of parametric model \\
$L$ & Terminal value in state $S_{t}$, where $S_{t} \in\left\{S_{t}^{\prime} \in \mathcal{S}_{t}: b^{D}\left(N_{t}^{\prime}\right)=\emptyset\right\}$ \\
$G_{t}^{T}\left(S_{t}\right)$ & A lower bound on the continuation value at time $t$ given that we are \\
$\tilde{\Phi}_{t}\left(R_{t}, a_{t}\right)$ & in resource state $R_{t}$ and take action $a_{t}$ \\
& $\quad$ Variables \\
\hline
\end{tabular}




\begin{tabular}{ll}
\hline$S_{t}$ & State at time $t \in \mathcal{T}$ \\
$R_{t}$ & Resource state variable \\
$I_{t}$ & Information state variable, so that $S_{t}=\left(R_{t}, I_{t}\right)$ \\
$a_{t}$ & Action (or decision) at time $t$ \\
$a_{t h}$ & (Binary) decision at time $t$ for transition $h$, so that $a_{t}=\left(a_{t h}\right)_{h \in b^{D}\left(N_{t}\right)}$ \\
$\mathcal{A}_{S_{t}}$ & Feasible region when in $S_{t}$ at time $t$ \\
$\alpha_{l}\left(S^{R}\left(R_{t}, a_{t}\right)\right)$ & Regression coefficient (or weight) when we are in $R_{t}$ and take action $a_{t}$ \\
$W_{t}$ & Exogenous information that first becomes known at time $t$ \\
\hline & \multicolumn{1}{c}{ Functions and mappings } \\
\hline$b^{D}\left(N_{t}\right)$ & Set of outgoing transitions of node $N_{t}$ \\
$S^{M}\left(S_{t}, a_{t}, W_{t+\Delta_{h}}\right)$ & Transition function, giving state $S_{t+\Delta_{h}}$ given that we are in state $S_{t}$, \\
& take action $a_{t}$ (i.e. make transition $\left.h\right)$, and then learn $W_{t+\Delta_{h}}$, which \\
$S^{R}\left(R_{t}, a_{t}\right)$ & is revealed between $t$ and $t+\Delta_{h}$ \\
& Resource transition function, giving resource state $R_{t+\Delta_{h}}$ given that \\
$\Pi_{t}\left(S_{t}, a_{t}\right)$ & we are in resource state $R_{t}$ and take action $a_{t}($ i.e. make transition $h)$ \\
$G_{t}\left(S_{t}\right)$ & Payoff at time $t$ given we are in state $S_{t}$ and take action $a_{t}$ \\
$\bar{G}_{t}\left(S_{t}\right)$ & Value of portfolio of real options when in state $S_{t}$ at time $t$ \\
$\hat{\Phi}_{t}^{L}\left(S_{t}, a_{t}\right)$ & Approximation of $G_{t}\left(S_{t}\right)$ \\
& Approximation of the continuation function at time $t$ when in state $S_{t}$ \\
\hline
\end{tabular}

\section{Appendix C. Effect of parametric model choices on portfolio value}

In this appendix we investigate the effect of different parametric models on the value of the copper mine investment project. In particular, we study the Power series, Laguerre, Hermite, Legendre and generalized Chebyshev polynomials of degree $L$, with $L \in\{1,2,3,4,5,6\}$. Considering the complete set of polynomials as described by Judd (1998) and the families of orthogonal polynomials as defined in Abramowitz and Stegun, 1972), as well as using the parameters of Table C.4. Table C.5 shows the portfolio value for different univariate orthogonal polynomials and different dimensions, $L$, of the parametric model. Independent of the chosen family of polynomials, since we have four

Table C.4: Parameters of convenience yield process for different specifications of Tsekrekos et al. (2012).

\begin{tabular}{lcccc}
\hline Spec. & $\rho_{x, \delta}$ & $\theta_{\delta}=\delta_{0}$ & $\sigma_{\delta}$ & $\kappa_{\delta}$ \\
\hline 1 & 0.40 & 0.01 & 0.05 & 0.30 \\
41 & 0.60 & 0.10 & 0.10 & 0.50 \\
81 & 0.80 & 0.15 & 0.15 & 0.80 \\
\hline
\end{tabular}


Table C.5: Value of investment project with portfolio of options (in $\$$ millions) for the specifications of Table C.4 and different parametric models (Standard error in parentheses).

\begin{tabular}{|c|c|c|c|c|c|c|}
\hline \multicolumn{7}{|c|}{ Panel A: Power series } \\
\hline Spec. & $L=1$ & $L=2$ & $L=3$ & $L=4$ & $L=5$ & $L=6$ \\
\hline 1 & $10.385(0.083)$ & $10.742(0.096)$ & $10.858(0.107)$ & $10.993(0.118)$ & $11.091(0.126)$ & $11.201(0.131)$ \\
\hline 41 & $0.546(0.016)$ & $0.602(0.016)$ & $0.637(0.017)$ & $0.650(0.017)$ & $0.655(0.017)$ & $0.661(0.017)$ \\
\hline 81 & $0.002(0.001)$ & $0.020(0.003)$ & $0.020(0.003)$ & $0.023(0.003)$ & $0.024(0.003)$ & $0.026(0.003)$ \\
\hline \multicolumn{7}{|c|}{ Panel B: Laguerre } \\
\hline Spec. & $L=1$ & $L=2$ & $L=3$ & $L=4$ & $L=5$ & $L=6$ \\
\hline 1 & $10.385(0.083)$ & $10.742(0.096)$ & $10.870(0.107)$ & $10.988(0.117)$ & $11.098(0.124)$ & $11.241(0.133)$ \\
\hline 41 & $0.546(0.016)$ & $0.602(0.016)$ & $0.637(0.017)$ & $0.650(0.017)$ & $0.654(0.017)$ & $0.661(0.017)$ \\
\hline 81 & $0.002(0.001)$ & $0.020(0.003)$ & $0.020(0.003)$ & $0.023(0.003)$ & $0.024(0.003)$ & $0.026(0.003)$ \\
\hline \multicolumn{7}{|c|}{ Panel C: Hermite } \\
\hline Spec. & $L=1$ & $L=2$ & $L=3$ & $L=4$ & $L=5$ & $L=6$ \\
\hline 1 & $10.385(0.083)$ & $10.742(0.096)$ & $10.858(0.107)$ & $10.996(0.117)$ & $11.097(0.126)$ & $11.174(0.129)$ \\
\hline 41 & $0.546(0.016)$ & $0.602(0.016)$ & $0.637(0.017)$ & $0.650(0.017)$ & $0.655(0.017)$ & $0.661(0.017)$ \\
\hline 81 & $0.002(0.001)$ & $0.020(0.003)$ & $0.020(0.003)$ & $0.023(0.003)$ & $0.024(0.003)$ & $0.026(0.003)$ \\
\hline \multicolumn{7}{|c|}{ Panel D: Legendre } \\
\hline Spec. & $L=1$ & $L=2$ & $L=3$ & $L=4$ & $L=5$ & $L=6$ \\
\hline 1 & $10.385(0.083)$ & $10.742(0.096)$ & $10.858(0.107)$ & $10.997(0.118)$ & $11.106(0.123)$ & $11.181(0.129)$ \\
\hline 41 & $0.546(0.016)$ & $0.602(0.016)$ & $0.637(0.017)$ & $0.649(0.017)$ & $0.654(0.017)$ & $0.662(0.017)$ \\
\hline 81 & $0.002(0.001)$ & $0.020(0.003)$ & $0.020(0.003)$ & $0.023(0.003)$ & $0.024(0.003)$ & $0.026(0.003)$ \\
\hline \multicolumn{7}{|c|}{ Panel E: Gen. Cheyshev } \\
\hline Spec. & $L=1$ & $L=2$ & $L=3$ & $L=4$ & $L=5$ & $L=6$ \\
\hline 1 & $10.385(0.083)$ & $10.742(0.096)$ & $10.870(0.107)$ & $10.992(0.116)$ & $11.074(0.123)$ & $11.158(0.128)$ \\
\hline 41 & $0.546(0.016)$ & $0.602(0.016)$ & $0.637(0.017)$ & $0.650(0.017)$ & $0.655(0.017)$ & $0.661(0.017)$ \\
\hline 81 & $0.002(0.001)$ & $0.020(0.003)$ & $0.020(0.003)$ & $0.023(0.003)$ & $0.024(0.003)$ & $0.026(0.003)$ \\
\hline
\end{tabular}

stochastic factors, setting $L$ to $1,2,3,4,5$, and 6 , results in $5,15,35,70,126$, and 210 , respectively, basis functions (or regressors) in the respective parametric model.

It is evident from the results that in general the approximated value of the mine investment project (which is a lower bound on its true value) improved as the parametric model's dimension, $L$, increased, regardless of the family of polynomials used. This is in line with the multidimensional convergence results of Moreno and Navas (2003); Stentoft (2004b); Areal et al. (2008). As can be seen, the results for specifications 41 and 81 are essentially the same across polynomial families, suggesting that any polynomial family 
with sufficient degree $L$ can be used in these two cases. In the case of specification 1 , however, our analysis revealed considerable value differences amongst different polynomials for high model dimensions, with differences between the highest and lowest project values at around $\$ 32 \mathrm{k}$ and $\$ 83 \mathrm{k}$ for $L=5$ and $L=6$, respectively. This suggests that the approximation can be improved considerably by choosing an appropriate polynomial family. Note that while standard errors in specification 1 are higher than in the other two specifications, project values are proportionally even higher.

Compared with values obtained for specifications 41 and 81, which eventually levelled off at high $L$, the trends corresponding with specification 1 seem to indicate that values have not converged yet. This suggests that an increase in $L$ would further improve the lower bound. Although the Power series was found to perform surprisingly well and reduced computational time by about 40-45\% (which is similar to the reduction found by Areal et al. (2008)), the number of basis functions used in the regression grows exponentially in $L$. This substantially increased both the complexity of the parametric model and computational cost; e.g., plus 84 basis functions and $50 \%$ more time when L is increased from 5 to 6 . In Section 5 , complete sets of Legendre and Hermite polynomials with $L=4$ are used for Table 2 and Figure 2, respectively. Although different parametric model choices may result in better approximations, our choice, which presents the best trade-off between accuracy and computational time amongst all models tested, is sufficient for the purposes of demonstration. Future work might therefore investigate the convergence properties and the computational efficiency of different parametric models in situations with both complex and large portfolios of interdependent real options.

\section{References}

Abramowitz, M., Stegun, I. A. (Eds.), December 1972. Handbook of Mathematical Functions: With Formulas, Graphs, and Mathematical Tables. Vol. 55 of Applied Mathematics Series. National Bureau of Standards, Washington, D.C., USA, 10th Printing.

Ahmed, S., King, A. J., Parija, G., 2003. A multi-stage stochastic integer programming approach for capacity expansion under uncertainty. Journal of Global Optimization $26(1), 3-24$.

Anand, J., Oriani, R., Vassolo, R. S., 2007. Managing a portfolio of real options. In: Reuer, J. J., Tong, T. W. (Eds.), Real Options Theory: Advances in Strategic Management. Vol. 24. Elsevier Ltd, Ch. 10, pp. 275-303.

Areal, N., Rodrigues, A., Armada, M. R., 2008. On improving the least squares Monte Carlo option valuation method. Review of Derivatives Research 11 (1-2), 119-151. 
Bellman, R., Dreyfus, S., 1959. Functional approximations and dynamic programming. Mathematical Tables and Other Aids to Computation 13 (68), 247-251.

Bendall, H. B., Stent, A. F., 2007. Maritime investment strategies with a portfolio of real options. Maritime Policy \& Management 34 (5), 441-452.

Bennouna, K., Meredith, G. G., Marchant, T., 2010. Improved capital budgeting decision making: evidence from Canada. Management Decision 48 (2), 225-247.

Block, S., 2007. Are "real options" actually used in the real world? The Engineering Economist 52 (3), 255-267.

Bowman, E. H., Hurry, D., 1993. Strategy through the option lens: An integrated view of resource investments and the incremental-choice process. The Academy of Management Review 18 (4), 760-782.

Brennan, M. J., Schwartz, E. S., 1985. Evaluating natural resource investments. The Journal of Business 58 (2), 135-157.

Brosch, R., 2008. Portfolios of Real Options. Vol. 611 of Lecture Notes in Economics and Mathematical Systems. Springer-Verlag Berlin Heidelberg.

Carriere, J. F., 1996. Valuation of the early-exercise price for options using simulations and nonparametric regression. Insurance: Mathematics and Economics 19 (1), 19 30 .

Charnes, J. M., Shenoy, P. P., 2004. Multistage Monte Carlo method for solving influence diagrams using local computation. Management Science 50 (3), 405-418.

Chevalier-Roignant, B., Flath, C. M., Huchzermeier, A., Trigeorgis, L., 2011. Strategic investment under uncertainty: A synthesis. European Journal of Operational Research $215(3), 639-650$.

Childs, P. D., Ott, S. H., Triantis, A. J., 9 1998. Capital budgeting for interrelated projects: A real options approach. Journal of Financial and Quantitative Analysis $33(3), 305-334$.

Clément, E., Lamberton, D., Protter, P., 2002. An analysis of a least squares regression method for American option pricing. Finance and Stochastics 6 (4), 449-471.

Cortazar, G., Casassus, J., 1998. Optimal timing of a mine expansion: Implementing a real options model. The Quarterly Review of Economics and Finance 38 (3), 755 769 .

Cortazar, G., Gravet, M., Urzua, J., 2008. The valuation of multidimensional american real options using the (LSM) simulation method. Computers \& Operations Research $35(1), 113-129$. 
De Reyck, B., Degraeve, Z., Vandenborre, R., 2008. Project options valuation with net present value and decision tree analysis. European Journal of Operational Research $184(1), 341-355$.

Demirer, R., Charnes, J. M., Kellogg, D., May 2003. Influence diagrams for real options valuation, available at: http://www.siue.edu/business/economicsandfinance/ pdf/demirer-agouron.pdf.

Denault, M., Simonato, J.-G., 2017. Dynamic portfolio choices by simulation-andregression: Revisiting the issue of value function vs portfolio weight recursions. Computers \& Operations Research 79, $174-189$.

Denault, M., Simonato, J.-G., Stentoft, L., 2013. A simulation-and-regression approach for stochastic dynamic programs with endogenous state variables. Computers \& Operations Research 40 (11), 2760 - 2769.

Gamba, A., December 2003. Real options valuation: A Monte Carlo approach. Working Paper 2002/3, Faculty of Management, University of Calgary.

Glasserman, P., 2003. Monte Carlo Methods in Financial Engineering, 1st Edition. Vol. 53 of Applications of mathematics. Springer-Verlag New York.

Haugh, M. B., Kogan, L., 2007. Duality theory and approximate dynamic programming for pricing american options and portfolio optimization. Handbooks in Operations Research and Management Science 15, 925 - 948.

Howard, R. A., Matheson, J. E., 2005. Influence diagrams. Decision Analysis 2 (3), $127-143$.

Judd, K. L., 1998. Numerical Methods in Economics. The MIT Press.

Kohler, M., 2010. A review on regression-based monte carlo methods for pricing american options. In: Devroye, L., Karasözen, B., Kohler, M., Korn, R. (Eds.), Recent Developments in Applied Probability and Statistics. Physica-Verlag HD, pp. 37-58.

Lander, D. M., Pinches, G. E., 1998. Challenges to the practical implementation of modeling and valuing real options. The Quarterly Review of Economics and Finance $38(3), 537-567$.

Lander, D. M., Shenoy, P. P., 1999. Modeling and valuing real options using influence diagrams. Working Paper 283, School of Business, University of Kansas, available at: http://pshenoy.faculty.ku.edu/Papers/WP283.pdf.

Longstaff, F. A., Schwartz, E. S., 2001. Valuing American options by simulation: a simple least-squares approach. Review of Financial Studies 14 (1), 113-147.

Loureiro, M. V., Claro, J., Pereira, P. J., 2015. Capacity expansion in transmission networks using portfolios of real options. International Journal of Electrical Power \& Energy Systems 64, $439-446$. 
Luehrman, T. A., 1998. Strategy as a portfolio of real options. Harvard business review $76,89-101$.

McGrath, R. G., Nerkar, A., 2004. Real options reasoning and a new look at the R\&D investment strategies of pharmaceutical firms. Strategic Management Journal 25 (1), $1-21$.

Meier, H., Christofides, N., Salkin, G., 2001. Capital budgeting under uncertainty an integrated approach using contingent claims analysis and integer programming. Operations Research 49 (2), 196-206.

Metcalf, G. E., Hassett, K. A., 1995. Investment under alternative return assumptions comparing random walks and mean reversion. Journal of Economic Dynamics and Control 19 (8), $1471-1488$.

Moreno, M., Navas, J. F., 2003. On the robustness of least-squares Monte Carlo (LSM) for pricing american derivatives. Review of Derivatives Research 6 (2), 107-128.

Myers, S. C., 1974. Interactions of corporate financing and investment decisionsimplications for capital budgeting. The Journal of Finance 29 (1), 1-25.

Myers, S. C., January-February 1984. Finance theory and financial strategy. Interfaces $14(1), 126-137$.

Nadarajah, S., Margot, F., Secomandi, N., 2017. Comparison of least squares monte carlo methods with applications to energy real options. European Journal of Operational Research 256 (1), $196-204$.

Pendharkar, P. C., 2010. Valuing interdependent multi-stage IT investments: A real options approach. European Journal of Operational Research 201 (3), 847 - 859.

Pizzi, C., Pellizzari, P., 2002. Monte carlo pricing of american options using nonparametric regression. Rendiconti per gli Studi Economici Quantitativi 1, 75-91.

Powell, W. B., 2011. Approximate Dynamic Programming: Solving the Curses of Dimensionality, 2nd Edition. John Wiley \& Sons, Inc.

Pringles, R., Olsina, F., Garcs, F., 2015. Real option valuation of power transmission investments by stochastic simulation. Energy Economics 47 (0), $215-226$.

Rose, S., 1998. Valuation of interacting real options in a tollroad infrastructure project. The Quarterly Review of Economics and Finance 38 (3), 711 - 723.

Ryan, P. A., Ryan, G. P., 2002. Capital budgeting practices of the Fortune 1000: How have things changed? Journal of Business and Management 8 (4), 355-364.

Sabour, S. A. A., Poulin, R., 2006. Valuing real capital investments using the leastsquares Monte Carlo method. The Engineering Economist 51 (2), 141-160. 
Savolainen, J., 2016. Real options in metal mining project valuation: Review of literature. Resources Policy 50, $49-65$.

Schwartz, E. S., 1997. The stochastic behavior of commodity prices: Implications for valuation and hedging. The Journal of Finance 52 (3), 923-973.

Sick, G., Gamba, A., 2010. Some important issues involving real options: an overview. Multinational Finance Journal 14 (1/2), 73-123.

Slade, M. E., 2001. Valuing managerial flexibility: An application of real-option theory to mining investments. Journal of Environmental Economics and Management 41 (2), $193-233$.

Smit, H. T. J., Trigeorgis, L., 2006. Strategic planning: valuing and managing portfolios of real options. R\&D Management 36 (4), 403-419.

Smith, J. L., Thompson, R., 2008. Managing a portfolio of real options: Sequential exploration of dependent prospects. The Energy Journal 29, 43-61.

Stentoft, L., 2004a. Assessing the least squares Monte-Carlo approach to American option valuation. Review of Derivatives Research 7 (2), 129-168.

Stentoft, L., 2004b. Convergence of the least squares Monte Carlo approach to American option valuation. Management Science 50 (9), 1193-1203.

Stentoft, L., 2014. Value function approximation or stopping time approximation: A comparison of two recent numerical methods for American option pricing using simulation and regression. Journal of Computational Finance 18 (1), 65-120.

Trigeorgis, L., 1993. The nature of option interactions and the valuation of investments with multiple real options. The Journal of Financial and Quantitative Analysis 28 (1), $1-20$.

Trigeorgis, L., 1995. Real options: An overview. In: Trigeorgis, L. (Ed.), Real Options in Capital Investment: Models, Strategies, and Applications. Ch. 1, pp. 1-28.

Trigeorgis, L., 2005. Making use of real options simple: An overview and applications in flexible/modular decision making. The Engineering Economist 50 (1), 25-53.

Trigeorgis, L., Kasanen, E., 1991. An integrated options-based strategic planning and control model. Managerial Finance 17 (2/3), 16-28.

Tsekrekos, A. E., Shackleton, M. B., Wojakowski, R., 2012. Evaluating natural resource investments under different model dynamics: Managerial insights. European Financial Management 18 (4), 543-575.

Tsitsiklis, J., Van Roy, B., Jul 2001. Regression methods for pricing complex Americanstyle options. IEEE Transactions on Neural Networks 12 (4), 694-703. 
Tsitsiklis, J. N., Van Roy, B., 1999. Optimal stopping of markov processes: Hilbert space theory, approximation algorithms, and an application to pricing high-dimensional financial derivatives. IEEE Transactions on Automatic Control 44 (10), 1840-1851.

van Bekkum, S., Pennings, E., Smit, H. T. J., 2009. A real options perspective on R\&D portfolio diversification. Research Policy 38 (7), 1150 - 1158.

Vassolo, R. S., Anand, J., Folta, T. B., 2004. Non-additivity in portfolios of exploration activities: a real options-based analysis of equity alliances in biotechnology. Strategic Management Journal 25 (11), 1045-1061.

Verbeeten, F. H., 2006. Do organizations adopt sophisticated capital budgeting practices to deal with uncertainty in the investment decision?: A research note. Management Accounting Research 17 (1), $106-120$.

Wang, T., de Neufville, R., June 2004. Building real options into physical systems with stochastic mixed-integer programming. In: Proceedings of the 8th Annual International Real Options Conference. Montréal, Canada.

Williams, H. P., 2013. Model Building in Mathematical Programming, 5th Edition. John Wiley \& Sons.

Wolsey, L. A., 1998. Integer programming. Wiley-Interscience Series in Discrete Mathematics and Optimization. John Wiley \& Sons.

Zapata, J. C., Reklaitis, G. V., 2010. Valuation of project portfolios: An endogenously discounted method. European Journal of Operational Research 206 (3), 653 - 666.

Zhang, S. X., Babovic, V., 2011. An evolutionary real options framework for the design and management of projects and systems with complex real options and exercising conditions. Decision Support Systems 51 (1), $119-129$. 